\title{
Swarm intelligence, exact and matheuristic approaches for minimum weight directed dominating set problem
}

\author{
Mallikarjun Rao Nakkala ${ }^{\mathrm{a}, 1}$, Alok Singh ${ }^{\mathrm{a}, *}$, André Rossi ${ }^{\mathrm{b}}$ \\ a School of Computer and Information Sciences, University of Hyderabad, Hyderabad 500 046, Telangana, India \\ ${ }^{\mathrm{b}}$ Université Paris-Dauphine PSL, LAMSADE UMR CNRS 7243, Place du Maréchal de Lattre de Tassigny, 75775 Paris Cedex 16, France
}

\section{A R T I C L E I N F O}

\section{Keywords:}

Directed dominating set

Minimum weight directed dominating set

Artificial bee colony algorithm

Invasive weed optimization algorithm

Swarm intelligence

Matheuristic

\begin{abstract}
A B S T R A C T
There exist numerous heuristic and exact approaches in the literature for addressing minimum weight dominating set problem (MWDS) on vertex-weighted undirected graphs which is a well known $\mathcal{N} \mathcal{P}$-hard problem. However, little attention has been paid to its counterpart in vertex-weighted directed graphs called minimum weight directed dominating set problem (MWDDS) despite its use in modeling real-world applications involving directed interactions. As directed graphs can model undirected graphs, MWDDS can be considered as a generalization of MWDS, and hence, MWDDS is also $\mathcal{N} \mathcal{P}$-hard. In this paper, we present two approaches based on swarm intelligence, one approach based on integer linear programming (ILP) and one matheuristic approach to address the MWDDS. These approaches are the first approaches for MWDDS in their respective categories. One of our swarm intelligence approach is based on artificial be colony (ABC) algorithm, whereas the other is based on invasive weed optimization (IWO) algorithm. Both these approaches are hybridized with problem specific heuristics and a local search mechanism. We have evaluated the performance of our approaches on benchmark instances derived from the standard benchmark instances of MWDS. Computational results show the effectiveness of our approaches.
\end{abstract}

\section{Introduction}

Given a vertex-weighted directed graph $G=(V, A, w)$, where $V$ is the set of vertices, $A$ is the set of arcs, and $w: V \rightarrow \mathbb{R}_{\geq 0}$ is the weight function that assigns a positive weight to each vertex of $V$. Each $\operatorname{arc}(u, v) \in A$ is directed from vertex $u$ to vertex $v$. In the context of the arc $(u, v)$, the vertex $u$ is referred to as parent vertex or initial vertex or predecessor vertex, and the vertex $v$ is referred to as child vertex or terminal vertex or successor vertex. A directed dominating set on this graph is a subset of vertices such that each vertex of the graph is either a member of this subset or the terminal vertex of at least one arc whose initial vertex is a member of this subset. In other words, a directed dominating set $S \subseteq V$ is such that each vertex $v \notin S$ is child of at least one vertex belonging to $S$. A vertex that belongs to $S$ is referred to as a dominating vertex or a dominator, and a vertex that is not a member of $S$ is referred to as a dominated or a dominatee or a non-dominating vertex. The minimum directed dominating set problem (MDDS) consists in finding a directed dominating set on $G$ having minimum number of vertices. The minimum weight directed dominating set problem (MWDDS) is a generalization of MDDS and seeks a directed dominating set on $G$ with minimum sum of weights of its constituent vertices. As directed graphs can model undirected graphs, MDDS and MWDDS can be considered as generalizations of corresponding problems in undirected graphs, viz. minimum dominating set problem (MDS) and minimum weight dominating set problem (MWDDS) respectively, which are well known $\mathcal{N} \mathcal{P}$-hard problems (Garey and Johnson, 1979). Hence, MDDS and MWDDS are also $\mathcal{N} \mathcal{P}$-hard. Throughout this paper, we will use vertex and node, and, directed graph and digraph interchangeably.

The in-degree of a vertex $v$ is the number of its parent vertices, and the out-degree of a vertex $v$ is number of its child vertices. A source vertex is a vertex with in-degree zero, and a leaf vertex is a vertex with out-degree zero. An isolated vertex is a vertex with both in-degree and out-degree zero, i.e., an isolated vertex is both a source vertex and a leaf vertex. Obviously, all source vertices (including isolated vertices) must be part of each and every directed dominating sets.

MDDS and MWDDS are the smallest set of vertices and the smallest weighted set of vertices respectively that have directed edges to all other vertices of the digraph. Hence, they can be used at minimum cost to monitor and control the directed interactions in complex networks arising in diverse areas such as spread of infectious diseases (Takaguchi et al., 2014), power generation and transmission (Yang et al., 2012), chemical reaction and metabolic regulation (Liu et al., 2013), genetic

\footnotetext{
* Corresponding author.

E-mail addresses: alokcs@uohyd.ernet.in (A. Singh), andre.rossi@dauphine.psl.eu (A. Rossi).

1 Mallikarjun passed away on April 29, 2021 due to COVID-19. The second and third authors take full responsibility for the content of this paper.
} 
regulation (Wuchty, 2014; Wang et al., 2014). Concept of domination in digraphs has also been used for routing in ad hoc wireless networks with unidirectional links (Wu, 2002) and processing skyline queries in databases (Koltun and Papadimitriou, 2005). MDDS is used in case of homogeneous networks. On the other hand, MWDDS is used in case of networks composed of heterogeneous vertices where vertices differ in their capability/cost to monitor and control the directed interaction. Ghoshal et al. (1998) cited the application of MWDDS in facility location where a set of locations is given and each location has a positive weight representing the cost of opening the facility at that location. There is an arc from location $u$ to location $v$ if location $v$ can be reached from location $u$. Identical facilities need to be opened in a subset of these locations in such a manner that those locations which are not in this subset should be reachable from at least one member of the subset and total cost of opening all these facilities is minimized. Facilities in this example can be radio or TV stations. Obviously, this is an instance of MWDDS.

Minimum weight dominating set problem (MWDS) on undirected graphs is a widely-studied problem, and many approaches (Albuquerque and Vidal, 2018; Lin and Guan, 2018; Wang et al., 2018; Lin et al., 2016; Chaurasia and Singh, 2015a; Nitash and Singh, 2014; Potluri and Singh, 2013; Jovanovic et al., 2010; Shetgaonkar and Singh, 2020) have been proposed in the literature for this problem. However, the corresponding problem in directed graphs, viz. MWDDS is an understudied problem, though several approaches exist in the literature for MDDS (Pang et al., 2010; Molnár Jr. et al., 2013; Takaguchi et al., 2014; Habibulla et al., 2015). Nakkala and Singh (2020) presented five greedy heuristics to solve MWDDS. A local search mechanism is also presented to further improve the quality of solutions obtained through these greedy heuristics. These heuristics are the first heuristic approaches for MWDDS in the literature. A brief overview of these heuristics has been provided in Section 2. This problem has not been studied in the literature from the perspective of metaheuristics, exact approaches or matheuristics.

Motivated by the absence of any metaheuristic, integer linear programming (ILP) or matheuristic approach in the literature for solving MWDDS, we have developed two swarm intelligence based metaheuristic approaches, an exact approach based on ILP and a matheuristic approach for MWDDS. Our first swarm intelligence approach is based on artificial bee colony (ABC) algorithm, whereas the second approach is based on invasive weed optimization (IWO) algorithm. The ABC algorithm proposed by Karaboga (2005) upon getting inspiration from intelligent foraging behavior of honey bees has been successfully used to solve numerous optimization problems in various domains, e.g. Singh (2009), Singh and Sundar (2011), Chaurasia and Singh (2015b), Alshamiri et al. (2016), Jayalakshmi and Singh (2017), Pandiri and Singh (2018, 2019), Pandiri et al. (2020). Likewise, the invasive weed optimization (IWO) proposed by Mehrabian and Lucas (2006) is inspired by colonizing behavior exhibited by weeds in an ecosystem. IWO algorithm too has been applied to solve a number of optimization problems (Venkatesh and Singh, 2015; Alshamiri et al., 2018; Safari et al., 2020; Liu and Nie, 2021). Motivated by the effectiveness demonstrated by $\mathrm{ABC}$ and IWO algorithms in solving numerous optimization problems, we have developed $\mathrm{ABC}$ and IWO algorithms based approaches to solve the MWDDS problem. For small and medium size instances, an exact approach based on ILP may be used to address $\mathcal{N} \mathcal{P}$-hard optimization problems like MWDDS. It can also serve the purpose of assessing the quality of the solutions returned by metaheuristics for the instances that can be solved to optimality. This served as the motivation for developing an ILP based approach for MWDDS. In addition, a matheuristic algorithm that takes an approximate solution as an input and tries to improve it by exploring its neighborhood using ILP is also proposed for those instances that are out of the range of an exact approach. We have evaluated the performance of our approaches on benchmark instances derived from the standard benchmark instances available in the literature for MWDS. Computational results on these benchmark instances show the effectiveness of our approaches.
The rest of this paper is organized as follows: Section 2 provides a brief overview of five existing greedy heuristics for MWDDS. Our ABC and IWO approaches for the MWDDS problem have been described in Section 3 and Section 4 respectively. Section 5 presents our integer linear programming approach, whereas our matheuristic approach is described in Section 6. Section 7 provides the computational results and their analysis. Finally, Section 8 outlines some concluding remarks about contributions of this paper and possible directions for future research.

\section{Greedy heuristics}

There are only five greedy heuristics available for MWDDS in the literature (Nakkala and Singh, 2020). These heuristics are called H1, H2, H3, H4 and H5 in Nakkala and Singh (2020). As these heuristics have been utilized in our swarm intelligence approaches, we have provided a brief overview of their working in this section. One may refer to Nakkala and Singh (2020) for motivation behind each of these heuristics. These heuristics have been utilized in two ways in our swarm intelligence approaches, viz. to generate 5 initial solutions each corresponding to one heuristic and to generate neighboring solutions. For generating an initial solution, each of these heuristics begins with an empty solution, and for generating a neighboring solution begins with an incomplete solution containing some dominating nodes. Then each of these heuristics iteratively builds a directed dominating set where during each iteration a vertex is added to the incomplete solution by following a greedy policy which varies from heuristic to heuristic. These iterations continue till a directed dominating set is obtained, and then, an improve procedure is applied to further reduce the cardinality of the obtained directed dominating set. Algorithm 1 provides the pseudo-code for our heuristics where greedy_policy() is the only function whose implementation differ for each of $\mathrm{H} 1, \mathrm{H} 2, \mathrm{H} 3, \mathrm{H} 4$ and H5. For generating an initial solution, a heuristic $H i(i=1 \ldots 5)$ is called with $\mathrm{Hi}(G, \emptyset)$, and for generating a neighboring solution with $\operatorname{Hi}(G, S)$, where $S$ is the incomplete solution containing some dominating nodes.

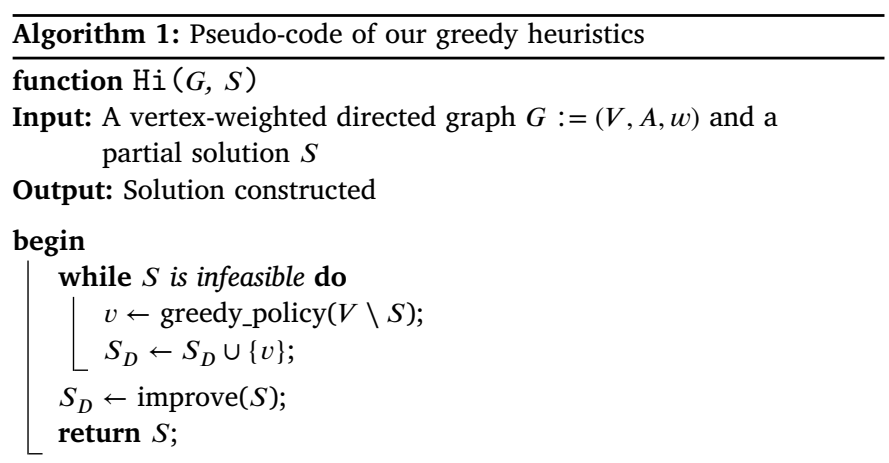

While constructing the directed dominating set, all those vertices which are either part of the partially constructed directed dominating set or have at least one parent vertex as part of the partially constructed directed dominating set are said to be covered, whereas all other vertices are said to be uncovered. As is the usual practice with dominating set problems, we have followed a coloring scheme to aid in description of working of these heuristics. Vertices with color white represent uncovered vertices, whereas the vertices colored black or gray are covered (dominating or dominated). Dominating vertices are represented with black, and the dominated vertices are represented with gray. Our heuristics work iteratively to construct a feasible solution. Initially, all vertices in the graph are assumed to be of white color. A non-black vertex is selected and colored black in every iteration, and all its white children are colored gray. This iterative process continues till no white vertex left in the graph. 
Table 1

Notations and their explanation.

Notation Explanation

$C W(u) \quad 1$ if vertex $u$ is of white color, 0 otherwise

$W_{w c}(u) \quad$ Sum of weights of white children of vertex $u$, i.e., $\sum_{(u, v) \in A} w(v) \times C W(v)$

$N_{w c}(u) \quad$ Number of white children of vertex $u$, i.e., $\sum_{(u, v) \in A} C W(v)$

$N_{p}(u) \quad$ Number of parents of vertex $u$

Before describing the greedy policy used by each of these heuristics, we will introduce few notational conventions in Table 1 and two equations based on these notational conventions.

$P_{\text {score }}(u) \leftarrow \frac{W_{w c}(u)+C W(u) \times w(u)}{w(u)}$

$T_{\text {score }}(u) \leftarrow \frac{N_{w c}(u)+C W(u)}{w(u)}$

Now, the greedy policy of each heuristic is described below:

- Heuristic H1: In every iteration, H1 tries to select a non-black vertex with the highest score as per (1) (highest $P_{\text {score }}$ ), a dominating vertex. If two or more vertices have the highest $P_{\text {score }}$, then from among these vertices, a vertex having the highest score as per (2) (highest $T_{\text {score }}$ ) is selected. If still there are more than one vertex having the highest $T_{\text {score }}$, then one such vertex is selected arbitrarily.

- Heuristic H2: H2 begins by making all source vertices as dominating vertices, and, then it proceeds exactly like $\mathrm{H} 1$

- Heuristic H3: H3 is same as H2 except in case of a tie as per (1) (highest $P_{\text {score }}$ ), it selects among the tied vertices, a white vertex with the minimum number of parents (minimum $N_{p}$ ). If still there are more than one white candidate then ties are broken arbitrarily. In case there is no white vertex among the vertices having the tie, then a gray node with the highest score is selected. If two or more gray nodes have the same highest score, then one of them is chosen uniformly at random.

- Heuristic H4: Like H2 and H3, this heuristic also selects all source vertices as dominating vertices at the beginning. After that, in every iteration, H4 makes use of following two rules while selecting a dominating vertex.

Rule 1: It is an extension of rule 2 in Habibulla et al. (2015) for computing minimum directed dominating set on unweighted directed graphs. It searches for a white vertex with only one nonblack parent and without any white child in increasing order of vertex ids and the search stops when first such vertex is found. If such a white vertex is found, then the white vertex or its nonblack parent will be selected as a dominating vertex depending on who among the two have the highest score as per (1). If they have the same score, then the non-black parent becomes the dominating vertex.

Rule 2: If rule 1 is unable to find a suitable vertex, then the dominating vertex is selected in the same way as in $\mathrm{H} 1$.

- Heuristic H5: H5 is the same as H4 except for rule 2. A vertex is selected exactly in the same way as in $\mathrm{H} 3$ when there is no source node.

\section{The ABC approach to MWDDS problem}

This section is devoted to our ABC algorithm based approach for MWDDS. We have combined our ABC approach with the problemspecific heuristics and a local search mechanism with the intention of providing high quality solutions. We will not provide any introduction to $\mathrm{ABC}$ algorithm as it is a well-established metaheuristic technique. However, those requiring such an introduction may refer to this recent survey (Akay et al., 2021).

Salient features of our ABC approach for MWDDS are described in the following subsections.

\subsection{Solution encoding and fitness}

The solution is represented directly by the set of dominating nodes. The objective function is used as the fitness function. As the MWDDS problem is a minimization problem, lower the objective function value of a solution, more fit it is considered.

\subsection{Generation of initial employed bee solutions}

The initial employed bee population has been generated in two ways. Initial five population members have been generated by each of the five heuristics discussed in the previous section. The remaining population members are generated by following an iterative procedure that is a mix of greediness and randomness. During each iteration of this procedure, with the probability $P_{r}$, a non-black vertex is randomly selected and added to the candidate solution. Otherwise, a node is added greedily. Here in the greedy procedure, to select a dominating node, every time one of the five heuristics is selected uniformly at random. This procedure continues until the candidate solution becomes a directed dominating set. We have utilized 5 greedy heuristics with the intention of generating superior quality diverse solutions.

\subsection{Procedure used by onlooker bees to select a food source}

The method of binary tournament selection (BTS) has been employed to select a food source for an onlooker bee. In this method, two food sources are selected randomly and their fitness values are compared. The better food source between the two is chosen with the probability $P_{o l}$ and the worst with probability $1-P_{o l}$.

\subsection{Neighboring solution generation}

During the employed bee phase, for every employed bee solution (population member), a neighboring solution is generated, and its fitness is compared with the corresponding employed bee solution. During onlooker bee phase, a neighboring solution is generated around only selected employed bee solutions and its fitness is compared with the corresponding employed bee solution. In both the phases (employed bee phase and onlooker bee phase), if the newly generated neighbor is more fit than the corresponding employed bee solution, then neighbor replaces the employed bee solution immediately, otherwise the employed bee solution is retained. To generate a neighboring solution for an employed bee solution, we have used a destroy-and-repair procedure (discussed in Section 3.4.1) and an improve procedure (discussed in Section 3.4.3). Destroy-and-repair procedure takes an employed bee solution $S$ as input and copies some vertices randomly from the solution $S$ to a solution $S^{\prime}$ which is initially empty. Obviously, $S^{\prime}$ will be infeasible. $S^{\prime}$ is then repaired, and the resulting feasible solution is passed to the improve procedure which further improves the solution quality. Pseudo-code for generating a neighboring solution has been given in Algorithm 2.

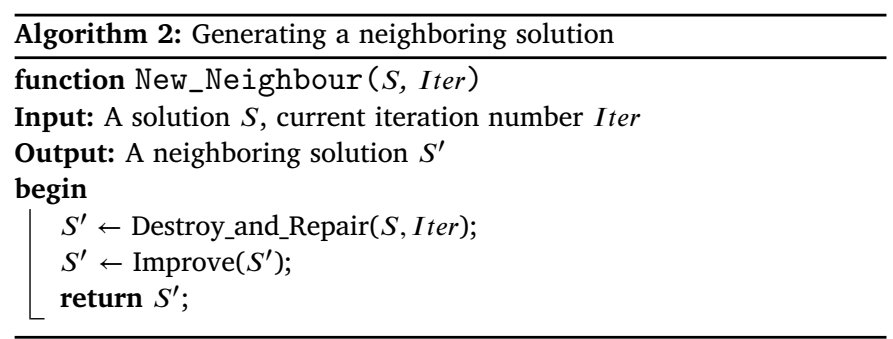

\subsubsection{Destroy-and-repair procedure}

This procedure takes an employed bee solution $S$ as input and iteratively copies $\lfloor R \times|S|\rfloor$ vertices randomly from the solution $S$ to 
an empty solution $S^{\prime}$, where $0<R<1$ is a parameter to be determined empirically. Clearly, $S^{\prime}$ will be infeasible. $S^{\prime}$ is then repaired into a feasible solution. Then this procedure tries to select the most appropriate heuristic among $\mathrm{H} 1-\mathrm{H} 5$ as per the procedure described in Section 3.4.2, so that infeasible solution can be transformed into a high quality solution. The selected heuristic iteratively adds vertices from $V \backslash S^{\prime}$ to $S^{\prime}$ as per its greedy policy. Iterations of the heuristic continue till the solution becomes feasible.

Algorithm 3 provides the pseudo-code of our destroy-and-repair procedure where $\operatorname{Random}(S)$ is a function that returns an element of set $S$ randomly, and, Random_Heuristic() is a function that selects a heuristic among $\mathrm{H} 1, \mathrm{H} 2, \mathrm{H} 3, \mathrm{H} 4$, and $\mathrm{H} 5$ uniformly at random and returns the index of the heuristic selected. Select_Heuristic() is another function that selects a heuristic based on their selection probabilities according to the procedure described in Section 3.4.2 and returns the index of the heuristic selected. The last function Update_Heuristic_Probabilities() updates the selection probabilities of five heuristics as per the description in Section 3.4.2.

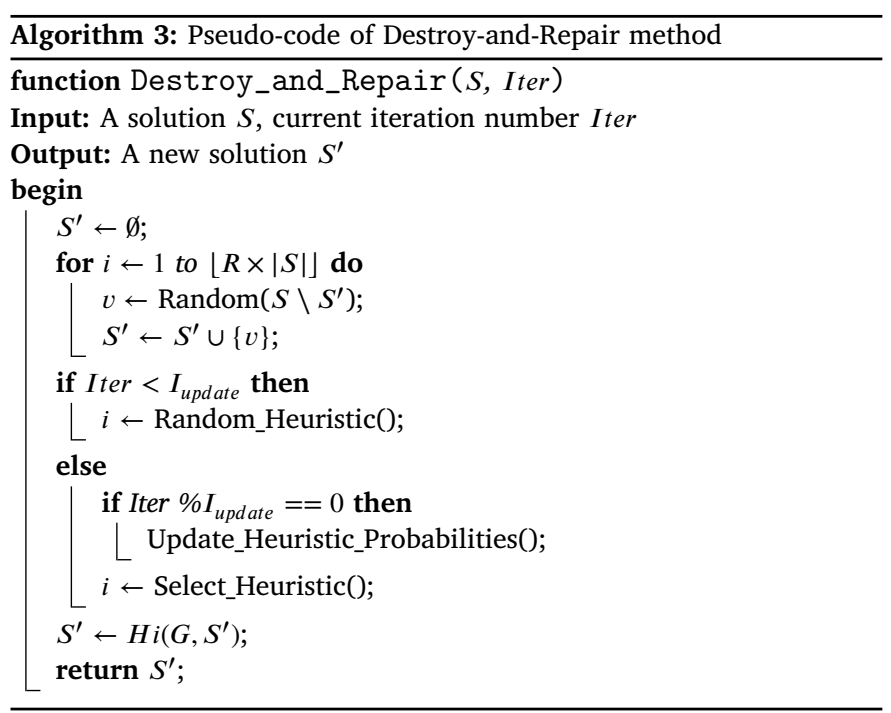

\subsubsection{Heuristic selection}

As the efficacy of different heuristics vary from one instance to another and even on the same instance from one phase in search process to another, we have developed an adaptive mechanism for selecting a heuristic. In this mechanism, each heuristic has a certain probability of selection that depends on its performance in the previous $I_{\text {update }}$ number of iterations. This selection probability is updated after every $I_{\text {update }}$ iterations.

In this mechanism, each heuristic has equal probability of selection up to $I_{\text {update }}$ initial iterations, and hence, one of the heuristics is selected uniformly at random. After $I_{\text {update }}$ initial iterations, each heuristic is selected according to its own probability of selection. Each heuristic $\mathrm{Hi}$ has a count $\mathrm{Hi}_{-}$used to keep track of the number of times that heuristic $\mathrm{Hi}$ got used, and, another count Hi_improved to keep track of the number of times, it has improved the corresponding employed bee solution. Heuristic probabilities are recomputed by using these two counts every $I_{\text {update }}$ number of iterations. Hi_used and Hi_improved are initialized to zero at the beginning and after every $I_{\text {update }}$ number of iterations after heuristic probabilities have been recomputed. Heuristic probabilities are computed using Eqs. (3) and (4). Eq. (3) computes $X_{i}$, which refers to the success rate of heuristic $H i$. The probability $P_{H i}$ of using a particular heuristic $H i$ is determined by using Eq. (4). Based on these probabilities, the five heuristics get selected for the next $I_{\text {update }}$ number of iterations after which these probabilities are computed again.

$X_{i}=\frac{\text { Hi_improved }}{\text { Hi_used }}$
$P_{H i}=\frac{X_{i}}{\sum_{j=1}^{5} X_{j}}$

\subsubsection{Improve method}

The solution obtained by destroy-and-repair procedure is further improved by using a local search mechanism. For each vertex, whether dominating or non-dominating, a reference count has been maintained that keeps track of number of dominating vertices covering it and this reference count is updated for all affected vertices whenever a vertex has been added to/removed from the set of dominating vertices. Note that a dominating vertex also cover itself. If the reference count of a dominating vertex and all its children is more than one, then that vertex can be removed from the solution without affecting the feasibility of the solution. Such dominating vertices are termed as redundant vertices. Our improve method begins by computing the set of redundant vertices and then iteratively removes the redundant vertices one-by-one till there is no redundant vertex. In each iteration, the redundant vertex having the highest ratio of its weight to out-degree is removed (ties are broken arbitrarily) and set of redundant vertices is computed again. Algorithm 4 provides the pseudo-code of our improve method where $\operatorname{Re} f(v)$ is the reference count of vertex $v$.

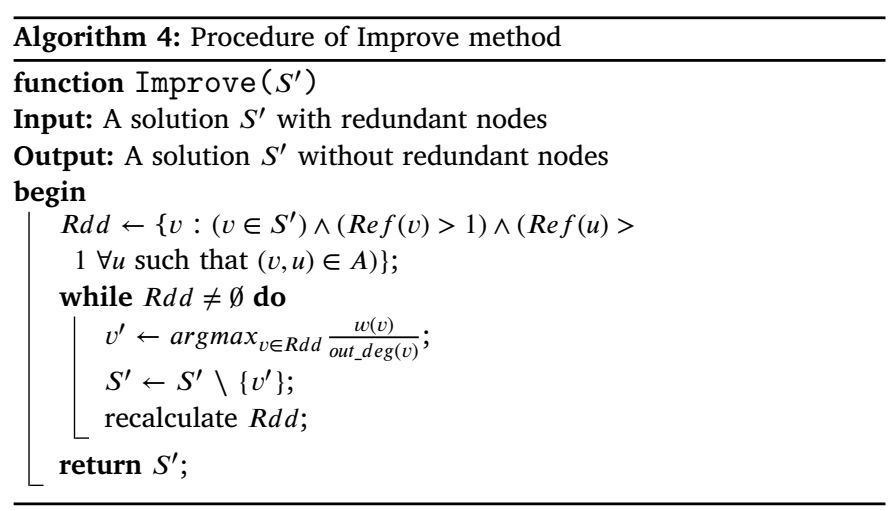

\subsection{Other features}

If an employed bee solution has not improved over a fixed number (limit $_{\text {non_imp }}$ ) of trials, where limit $_{\text {non_imp }}$ is a parameter to be determined empirically, then that employee bee solution is replaced with a new solution which is generated in the same manner as an initial solution other than first 5 initial solutions. Note that the number of trials for an employed bee solution means the number of times the employed bee solution is called for generating a neighboring solution. A solution may be called more than once in an iteration based on the number of onlookers choosing this solution.

Algorithm 5 provides the pseudo-code of our ABC approach where $E_{n}$ and $O_{n}$ are number of employed bees and onlooker bees. GR_initial_solution() is a function that generates an initial solution as per mixed greedy-random procedure described in Section 3.2 and New_Neighbour $\left(S_{i}\right)$ is a function that generates a solution in the neighborhood of solution $S_{i}$ as per procedure described in Section 3.4. $B T S\left(S_{1}, S_{2}, \ldots, S_{E_{n}}\right)$ is another function that selects and returns a solution from among solutions $S_{1}, S_{2}, \ldots, S_{E_{n}}$ as per binary tournament selection (Section 3.3).

\section{The IWO approach to MWDDS problem}

This section presents our IWO based approach for MWDDS. Like our $A B C$ approach, our IWO approach too makes use of the problemspecific heuristics and a local search mechanism. Our IWO approach has been designed by borrowing several features from ABC approach described in the previous section (Section 3 ). Solution encoding, fitness 


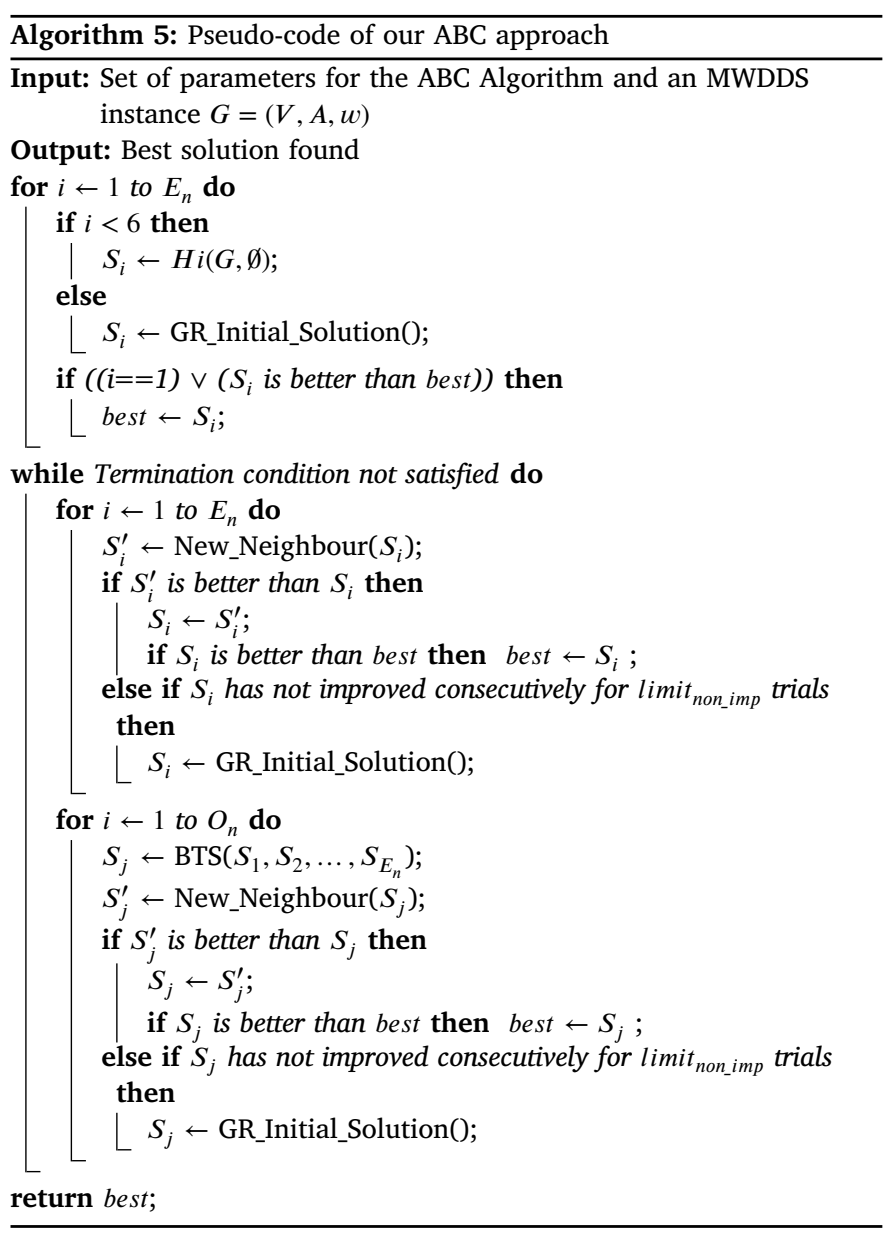

function, destroy-and-repair procedure, and improve method are the same as ABC. As IWO is not as well-established metaheuristic technique as $\mathrm{ABC}$ algorithm, we will provide a brief introduction to IWO prior to describing salient features of our IWO approach for MWDDS.

\subsection{A brief introduction to invasive weed optimization (IWO)}

Invasive weed optimization (IWO) is a recently proposed swarm intelligence algorithm inspired by the colonizing behavior of weeds. As the weeds have the ability to grow and multiply in an intrusive and rapid manner, they are considered detrimental to the growth of desirable, cultivated plants. Weeds have immense survival capacity owing to their ability to adopt as per their environment. Hence, weeds are always considered a serious threat to farming. IWO algorithm is proposed by Mehrabian and Lucas (2006).

In the IWO algorithm, each weed/weed plant represents a potential solution to the problem at hand and the fitness of a weed means the fitness of the solution that this weed represents. The IWO algorithm begins by creating an initial population of $n_{\text {init }}$ solutions (weed plants or weeds in short). These solutions are usually generated in a random manner. Then an iterative process ensues. In each iteration, certain number of seeds (child solutions) are created by each weed as per its own fitness. The number of seed solutions, a weed solution can create is usually mapped linearly from a given minimum value $x_{\min }$ to a given maximum value $x_{\max }$ as per its fitness. Each weed creates child seeds randomly in its vicinity by following a normal distribution with mean equal to zero, but varying variance, thereby yielding some sort of randomized local search in the vicinity of each weed plant. Exact method for creating seed solutions depends on the problem at hand. However, the standard deviation (SD), $\sigma$, of the normal distribution is reduced from a given initial value $\left(\sigma_{i v}\right)$ to a given final value $\left(\sigma_{f v}\right)$ over the iteration. Usually, the SD at an iteration iter is determined by using the following expression.

$\sigma_{i t e r}:=\left(\frac{\text { iter }_{m x}-\text { iter }}{\text { iter }_{m x}}\right)^{n l m}\left(\sigma_{i v}-\sigma_{f v}\right)+\sigma_{f v}$

Where $\sigma_{\text {iter }}$ is the standard deviation at the current iteration, iter $_{m x}$ is the maximum permissible number of iterations and $n l m$ is non-linear modulation index. This distribution guarantees that the probability of dropping a seed in a distant area reduces nonlinearly over iterations.

Once all weeds have created seed solutions, a phase called competitive exclusion phase begins. All newly created seeds become the weeds for this phase. All these newly created weeds are added to the weed population in case the number of weeds in the population is less than the maximum number of weeds permitted in the population $n_{\max }$. When the number of weeds in the population becomes $n_{\max }$, only the best $n_{\max }$ weeds according to their fitness among the existing ones and newly created ones are kept in the population and remaining weeds are discarded. After the competitive exclusion phase finishes, the next iteration of IWO algorithm starts. This entire process repeats as long as the termination condition remains unsatisfied. Please note that number of weeds in the initial population is $n_{\text {init }}$ and maximum possible number of weeds in the population is $n_{\max }$. Hence, we start with a smaller initial population and wait for the population to reach its maximum permissible limit through insertion of seed solutions over few initial iterations before competitive exclusion phase can start.

\subsection{Solution encoding and fitness}

IWO uses the same solution encoding and fitness function as our $\mathrm{ABC}$ approach (Section 3.1).

\subsection{Generation of initial weed solutions}

$n_{\text {init }}$ initial weed solutions are generated in the exactly same manner as the $\mathrm{ABC}$ initial employed bee solutions (Section 3.2).

\subsubsection{Determination of number of seeds a weed can produce}

Each weed solution in the population will produce a certain number of seed solutions (neighboring solutions). The number of seeds produced by a weed depends on its objective function value. The number of seeds produced by a weed is restricted to the closed interval of $\left\{x_{\min }\right.$, $\left.x_{\max }\right\}$. Weeds in the colony will be sorted in non-decreasing order of their objective function values. The colony is partitioned into $\left(x_{\max }-\right.$ $\left.x_{\min }\right)+1$ number of groups. Weeds in the first group will produce $x_{\max }$ number of seeds, and the second group of weeds will produce $x_{\max }-1$ number of seeds. Like this, the last group will produce $x_{\min }$ seeds. Algorithm 6 presents the procedure to determine the number of seeds a weed can produce where $n_{c}$ is the current population size. Please remember that MWDDS is a minimization problem and that is why weed solutions are sorted in non-decreasing order of their objective function values.

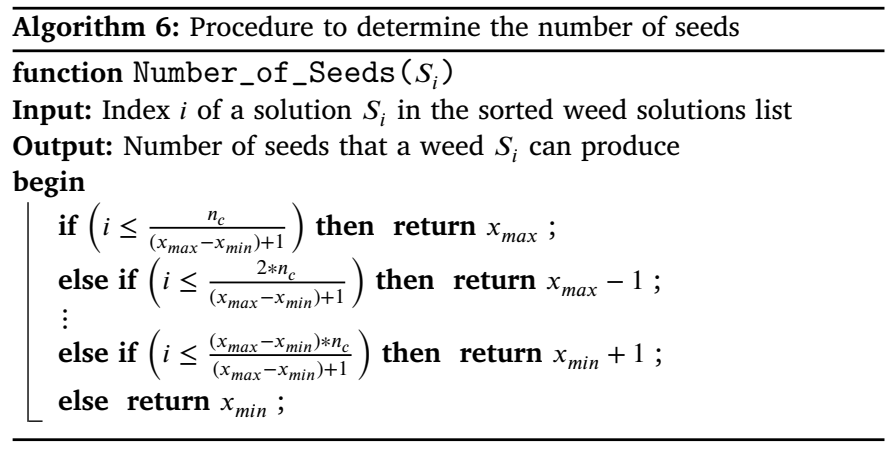




\subsubsection{Production of a seed by a weed}

A seed solution is generated in the same manner as a neighboring solution in our $\mathrm{ABC}$ approach, i.e., each seed solution is generated utilizing the destroy-and-repair procedure (discussed in Section 3.4.1) and the improve procedure (discussed in Section 3.4.3). Each seed solution produced by a weed is placed at the end of the population.

\subsection{Competitive exclusion}

This process has been used to eliminate inferior quality weed solutions so that only the superior quality weed solutions remain to produce seeds. In every iteration, when each weed solution in the population has produced its own seed solutions, the competitive exclusion phase begins. For this phase, all the newly produced seed solutions become weed solutions. Now, all the weed solutions (existing as well as newly produced) are sorted in non-decreasing order according to their objective function values. If the number of weed solutions in the population has exceeded the maximum allowed limit $n_{\max }$, then the excess weed solutions lying at the end of population are discarded.

Algorithm 7 provides the pseudo-code of our IWO approach where we have used the same function names as used in pseudo-code of our $\mathrm{ABC}$ approach for whatever components that we have borrowed from our ABC approach. In addition to these functions, Number_of_Seeds $\left(S_{i}\right)$ is a function that determines the number of seed solutions, a weed solution $S_{i}$ can produce and Sort_The_Weeds $\left(n_{c}\right)$ is a function that sorts all the weed solutions in population as per non-decreasing order of their objective function values.

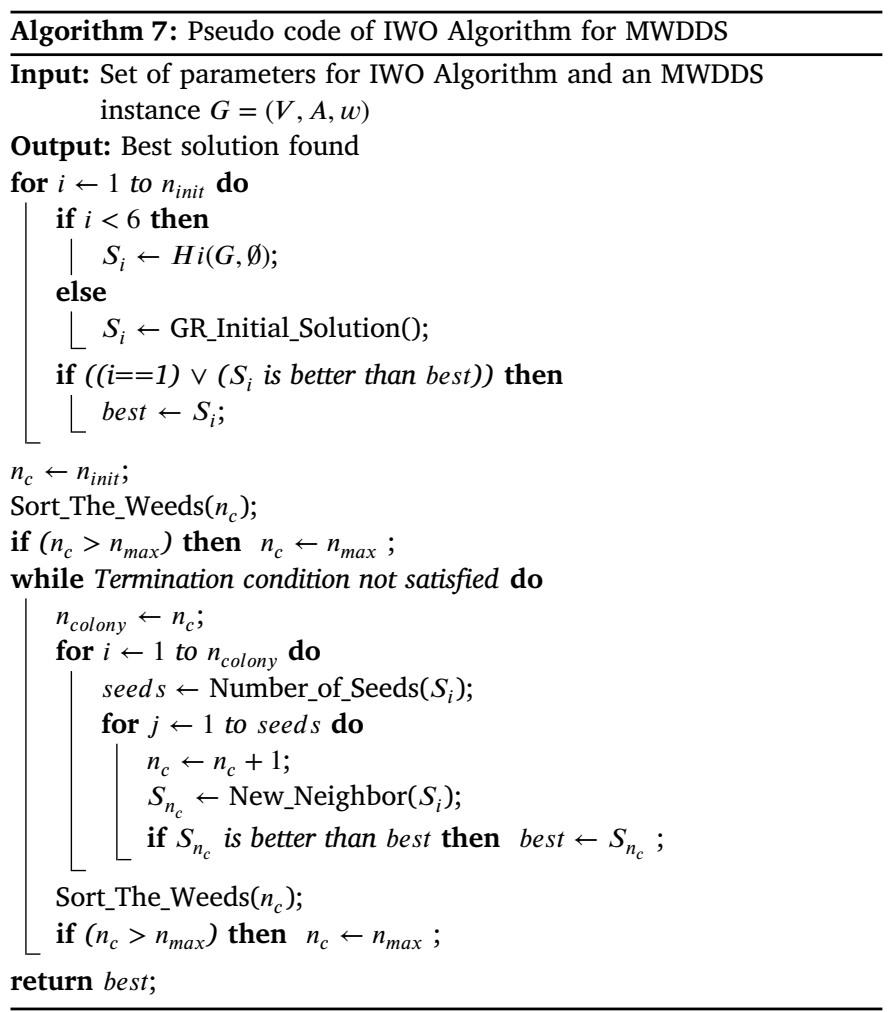

\section{Integer linear programming approach to MWDDS}

This section is devoted to our integer linear programming (ILP) approach for MWDDS. To formulate our ILP approach, we will make use of two sets $N_{G}^{+}\left(v_{i}\right)$ and $N_{G}^{-}\left(v_{i}\right)$ defined as follows: For all $v_{i} \in V$, $N_{G}^{+}\left(v_{i}\right)$ denotes the set of all the vertices $v_{j} \in V$ such that $(i, j)$ is an arc of $A$, and, $N_{G}^{-}\left(v_{i}\right)$ is the set of all the vertices $v_{j} \in V$ such that $(j, i) \in A$. Clearly in this notational convention, $\left|N_{G}^{+}\left(v_{i}\right)\right|$ and $\left|N_{G}^{-}\left(v_{i}\right)\right|$ denote the outdegree and indegree of a vertex $v_{i}$ respectively. In addition, $n=|V|$ is the order of the digraph $G$. In the sequel, we refer to our integer linear programming approach as ILP. We have used IBM CPLEX to solve the ILP.

\subsection{ILP model of MWDDS}

Let $x_{i}$ be the binary decision variable associated with vertex $v_{i} \in V$ : variable $x_{i}$ is set to one if and only if $v_{i}$ belongs to the minimum weight directed dominating set. The objective function of ILP is to minimize the total weight of the directed dominating set, and it has $n$ inequalities, that specify that each vertex $v_{i} \in V$ should be part of the minimum weight directed dominating set, or should have at least one parent that is part of this set. The remaining $n$ constraints are integrality constraints.

$$
\begin{array}{ll}
\text { Minimize } \sum_{v_{i} \in V} w_{i} x_{i} & \\
x_{i}+\sum_{v_{j} \in N_{G}^{-}\left(v_{i}\right)} x_{j} \geq 1 & \forall v_{i} \in V \\
x_{i} \in\{0,1\} & \forall v_{i} \in V
\end{array}
$$

This formulation is strengthened as follows. For each vertex $v_{i} \in V$, let $z\left(v_{i}\right)=1+\left|N_{G}^{+}\left(v_{i}\right)\right|>0$ be the maximum number of vertices that can be covered by vertex $v_{i}$. Since any solution to MWDDS should cover all the vertices, the following constraint holds: $\sum_{v_{i} \in V} z\left(v_{i}\right) x_{i} \geq n$. Let $Z$ be the set of all the pairwise different values of $z\left(v_{i}\right)$ (the cardinality of $Z$ is at most $n$ ). The previous constraint can be attempted for strengthening as follows:

$\sum_{v_{i} \in V}\left\lceil\frac{z\left(v_{i}\right)}{q}\right\rceil x_{i} \geq\left\lceil\frac{n}{q}\right\rceil \quad \forall q \in Z$

Since it is not guaranteed that one of these constraint dominates the original one, they are all enforced. If some of them are redundant, they can be removed at presolve stage by the ILP solver (CPLEX in our case).

These constraints can be shown useful by considering the circuit of order 3 , with $w(i)=1$ for all $v_{i} \in V$. The continuous relaxation of ILP yields 1.5 as the objective value, and a fractional solution $x_{i}=0.5$ for all $v_{i} \in V$. Adding the (unique) constraint above, which is simply $x_{1}+x_{2}+x_{3} \geq 2$ leads to an optimal solution to MWDDS.

\subsection{Graph reduction pre-process}

The following properties can be used as a pre-processing step to remove edges from the digraph $G$, and discard some vertices from the search of an optimal solution to MWDDS.

Property 0: If there exists $v_{i} \in V$ such that $N_{G}^{-}\left(v_{i}\right)$ is empty, then vertex $v_{i}$ must be part of any dominating set. As a consequence, the coverage requirement of all the vertices in $N_{G}^{+}\left(v_{i}\right)$ is satisfied, and all the arcs to $N_{G}^{+}\left(v_{i}\right)$ can be removed.

Property 1 (Strong vertices): If $v_{i}$ and $v_{j}$ are such that

$$
\begin{aligned}
& \text { - } w_{j} \leq w_{i} \\
& \text { - } N_{G}^{+}\left[v_{i}\right] \subseteq N_{G}^{+}\left(v_{j}\right)
\end{aligned}
$$

Then $v_{j}$ is stronger than $v_{i}$, and any solution of MWDDS containing $v_{i}$ can be turned into another one of lesser cost by deselecting $v_{i}$, and by selecting $v_{j}$ instead. As a consequence, $G$ can be modified by setting $w_{i}$ to $+\infty$, and by removing all the arcs of $N_{G}^{+}\left(v_{i}\right)$ since $v_{i}$ shall not dominate any vertex.

Property 2: If a vertex $v_{i} \in V$ is such that $w_{i} \geq \sum_{v_{j} \in N_{G}^{+}\left(v_{i}\right)} w_{j}$, and $N_{G}^{+}\left(v_{i}\right) \cap N_{G}^{-}\left(v_{i}\right) \neq \emptyset$ then $v_{i}$ does not appear in any optimal solution to MWDDS. Indeed, if it was present, it could be replaced by selecting all the vertices of $N_{G}^{+}\left(v_{i}\right)$ leading to a solution of lesser cost. In addition, since $N_{G}^{+}\left(v_{i}\right) \cap N_{G}^{-}\left(v_{i}\right) \neq \emptyset$, vertex $v_{i}$ is dominated. As a consequence, $G$ can be modified by setting $w_{i}$ to $+\infty$, and by removing all the arcs of $N_{G}^{+}\left(v_{i}\right)$ since $v_{i}$ shall not dominate any vertex. 


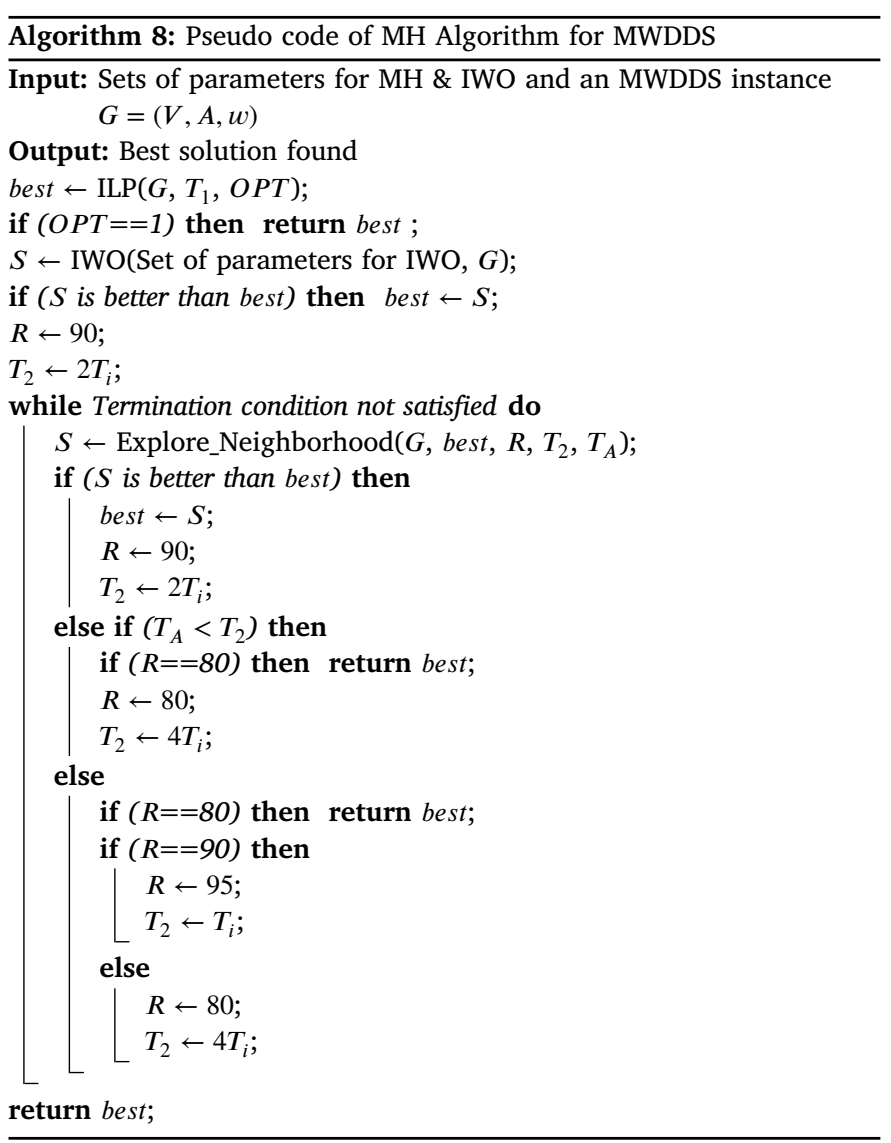

\section{Matheuristic approach to MWDDS}

We have also developed a matheuristic approach by combining our IWO approach with the ILP problem formulation described in the previous section. The reason for choosing IWO and not the ABC approach is the better performance of the former, in general, in comparison to the latter. We will refer to our matheuristic approach as $M H$.

Our matheuristic consists of solving the ILP formulation of previous section for $T_{1}$ amount of time. If ILP finds an optimal solution within this time then matheuristic stops. Otherwise IWO is executed and the best solution between the two solutions returned by ILP and IWO is chosen for further processing. We have used CPLEX to explore the neighborhood of the current solution, where the size of the solution space is controlled by the input parameter $R$ as follows: We enforce that at least $R \%$ of vertices from the existing solution must be part of the new solution, and let CPLEX find the best possible solution for $T_{2}$ seconds. This neighborhood exploration through CPLEX can be perceived as a local search in the context of our matheuristic. We have used this local search in an iterative framework, where value of $R$ varies over the set $\{80,90,95\}$ and the value of $T_{2}$ varies over the set $\left\{4 T_{i}, 2 T_{i}, T_{i}\right\}$. We start with $R=90$ and $T_{2}=2 T_{i}$ in the first iteration. With $R=90$ and $T_{2}=2 T_{i}$ in an iteration following are the possibilities:

- if the local search improves the solution then we retain this setting for the next iteration.

- If the local search terminates before $T_{2}$ time without improvement indicating no improved solution exists with this value of $R$, we use $R=80$ and $T_{2}=4 T_{i}$ in the next iteration.

- If the local search terminates at or after $T_{2}$ time without improvement thereby indicating value of $R$ may be too low for a meaningful exploration, we use $R=95$ and $T_{2}=T_{i}$ in the next iteration.
With $R=95$ and $T_{2}=T_{i}$ in an iteration, following are the possibilities:

- if the local search improves the solution then we move to $R=90$ and $T_{2}=2 T_{i}$.

- If the local search fails to improve the solution, we use $R=80$ and $T_{2}=4 T_{i}$ in the next iteration.

With $R=80$ and $T_{2}=4 T_{i}$ in an iteration, following are the possibilities:

- if the local search improves the solution then we move to $R=90$ and $T_{2}=2 T_{i}$.

- If the local search fails to improve the solution, matheuristic terminates.

Algorithm 8 provides the pseudo-code of $\mathrm{MH}$, where $\operatorname{ILP}\left(G, T_{1}\right.$, $O P T)$ is a function that solves the ILP formulation of previous section through CPLEX on MWDDS instance $G$ for $T_{1}$ amount of time and returns the solution found. It also sets the $O P T$ parameter to 1 if an optimal solution is found within this time, otherwise $O P T$ is set to zero. Explore_Neighborhood( $G$, best, $R, T_{2}, T_{A}$ ) is another function that explores the neighborhood of current solution best of instance $G$ through CPLEX for $T_{2}$ amount of time by retaining $R \%$ vertices of best in any solution that gets generated. It also returns the actual time taken in parameter $T_{A}$. IWO(Set of parameters for IWO, G) is yet another function implementing Algorithm 7.

\section{Computational results}

We have evaluated the performance of our approaches on three datasets, which we have derived from three standard datasets (TypeI, Type-II, and Unit disk graph (UDG)) available in the literature for MWDS on undirected graphs. The first two datasets, viz. Type-I and Type-II, are from Jovanovic et al. (2010). Both of these datasets have same graphs, but their vertex weights differ. For Type-I instances, vertices' weights are distributed uniformly at random in the closed interval of $[20,70]$, whereas for Type-II instances, vertices' weights are distributed in the interval $\left[1, \operatorname{degree}(v)^{2}\right]$, where degree $(v)$ is the degree of vertex $v$. In these datasets, the number of vertices varies from 50 to 1000 , and the number of edges varies from 50 to 20000 . There exist ten different instances for each combination of the number of vertices and the number of edges. Each of Type-I \& Type-II datasets has 530 instances. Third dataset UDG is from Potluri and Singh (2013), where vertices' weights are distributed randomly in the interval $[1,100]$. In this dataset, vertices are assumed to be distributed in an area of $1000 \times 1000$ uniformly at random, and any two vertices are considered connected by an edge if the distance between them is not exceeding a pre-specified range $R_{P}$. The number of vertices in this dataset is $\{50$, $100,250,500,750,1000\}$. Two values are considered for range $\left(R_{P}\right)$, viz. 150 and 200. There are ten instances for each combination of $R$ and the number of nodes. So there are 120 instances in the UDG dataset. Hence, a total of 1180 instances are there in these three datasets for MWDS.

We have derived the datasets for MWDDS from the corresponding datasets of MWDS by following the same process as described in Habibulla et al. (2015). In general, for every edge $\{u, v\}$ in an undirected graph, there are two arcs or directed edges $(u, v)$ and $(v, u)$ in the corresponding directed graph. Therefore, in order to transform an instance of MWDS to an instance of MWDDS, for each edge in the MWDS instance, we have selected randomly one of the two corresponding arcs, and, included it into the set of arcs for the instance of MWDDS. No change is made in weights of vertices when an MWDS instance is transformed into an MWDDS instance. These datasets for MWDDS will also be referred to as Type I, Type II and UDG.

Presented approaches are implemented in $\mathrm{C}$ and executed on Ubuntu based $3.6 \mathrm{GHz}$ Core-i7-7700 system with $32 \mathrm{~GB}$ of RAM. IBM CPLEX 12.7 library is used to solve the ILP model. None of our approaches 
Table 2

Comparison of H1, H2, H3, H4, H5, ABC, IWO, ILP, and MH on UDG dataset.

\begin{tabular}{|c|c|c|c|c|c|c|c|c|c|c|c|c|c|c|}
\hline$|V|$ & Range & H1 & $\mathrm{H} 2$ & H3 & $\mathrm{H} 4$ & H5 & $\mathrm{ABC}$ & $\mathrm{ABC}_{E T}$ & IWO & $\mathrm{IWO}_{E T}$ & ILP & $\mathrm{ILP}_{E T}$ & $\mathrm{MH}$ & $\mathrm{MH}_{E T}$ \\
\hline 50 & 150 & 820.0 & 818.1 & 818.1 & 828.0 & 828.0 & 805.2 & 1.9 & 805.2 & 1.9 & 805.2 & 0.0 & 805.2 & 0.0 \\
\hline 50 & 200 & 588.1 & 588.1 & 588.1 & 605.3 & 605.3 & 573.5 & 2.2 & 573.5 & 2.6 & 573.5 & 0.0 & 573.5 & 0.0 \\
\hline 100 & 150 & 1089.8 & 1091.9 & 1091.9 & 1111.3 & 1111.3 & 1065.8 & 6.9 & 1065.8 & 7.2 & 1065.8 & 0.0 & 1065.8 & 0.0 \\
\hline 100 & 200 & 642.8 & 641.0 & 641.0 & 644.7 & 644.7 & 614.6 & 8.3 & 613.8 & 8.4 & 613.7 & 0.0 & 613.7 & 0.0 \\
\hline 250 & 150 & 1025.2 & 1025.2 & 1024.1 & 1025.2 & 1024.1 & 985.4 & 32.4 & 985.1 & 31.1 & 984.1 & 0.0 & 984.1 & 0.0 \\
\hline 250 & 200 & 517.2 & 517.2 & 517.2 & 517.2 & 517.2 & 492.8 & 42.4 & 493.4 & 38.2 & 490.4 & 0.0 & 490.4 & 0.0 \\
\hline 500 & 150 & 856.9 & 856.9 & 855.1 & 856.9 & 855.1 & 814.8 & 115.0 & 814.9 & 101.9 & 813.2 & 0.1 & 813.2 & 0.1 \\
\hline 500 & 200 & 375.3 & 375.3 & 374.8 & 375.3 & 374.8 & 356.8 & 134.0 & 356.3 & 126.1 & 354.2 & 0.1 & 354.2 & 0.1 \\
\hline 800 & 150 & 659.0 & 659.0 & 659.0 & 659.0 & 659.0 & 625.5 & 271.7 & 624.3 & 242.9 & 619.9 & 0.3 & 619.9 & 0.3 \\
\hline 800 & 200 & 302.5 & 302.5 & 302.5 & 302.5 & 302.5 & 288.2 & 342.5 & 286.9 & 307.4 & 286.3 & 0.4 & 286.3 & 0.4 \\
\hline 1000 & 150 & 665.2 & 665.2 & 665.7 & 665.2 & 665.7 & 612.4 & 384.1 & 611.5 & 347.7 & 608.7 & 2.4 & 608.7 & 2.4 \\
\hline 1000 & 200 & 290.2 & 290.2 & 291.0 & 290.2 & 291.0 & 276.8 & 504.9 & 276.7 & 478.4 & 275.9 & 1.0 & 275.9 & 1.0 \\
\hline Avg: & & 652.7 & 652.6 & 652.4 & 656.8 & 656.6 & 626.0 & 153.9 & 625.7 & 141.2 & 624.3 & 0.4 & 624.3 & 0.4 \\
\hline
\end{tabular}

use any multi-core features. Our approaches are executed once on each instance of the dataset. All the datasets have ten instances in a group for each combination of the same number of nodes $(|V|)$ and the same number of directed edges $(|A|)$ or the same range $(R)$. We have reported for each approach the average solution quality and the average execution time on each group of ten instances like the usual practice of reporting results in case of MWDS.

Parameter values for our $\mathrm{ABC}$ and IWO approaches are chosen empirically after a large number of trials. First, we will describe the $\mathrm{ABC}$ parameter values. The number of initial employee bees $E_{n}$ is fixed to 75 , the number of onlookers $O_{n}$ is set to 125 , limit $_{\text {non_imp }}$ is set to 600 . When an employed bee solution has not improved continuously over a limit $_{\text {non imp }}$ number of trials, then that becomes a scout. To select an onlooker, we have used the binary tournament selection mechanism. In this, the better fitness solution has been selected with the probability $P_{o l}$ set to 0.8 . In destroy-repair procedure, $R$ is set to 0.6 . In heuristic selection mechanism, after every $I_{\text {update }}$ iterations the heuristic probabilities are recomputed. This parameter is set to 8 . While the generation of the initial population, in the mixed randomgreedy procedure, a dominating node has been selected randomly with $P_{r}$ set to 0.3 . Our ABC algorithm terminates when best solution has not improved over 2500 iterations.

As far as IWO parameter values are concerned, the number of initial weed $n_{\text {init }}$ is set to 300 . The minimum $x_{\min }$ and maximum $x_{\max }$ number of seeds that a weed can produce are set to 1 and 3 respectively. The maximum number of weeds allowed in the population $n_{\max }$ is set to 350. Same values of $R$ and $P_{r}$ as in ABC are used in IWO also, i.e., $R$ $=0.6$ and $p_{r}=0.3$. $I_{\text {update }}$ is set to 5 . IWO algorithm terminates when best solution has not improved over 750 iterations.

ILP and MH approaches were allowed to execute for a maximum of $3600 \mathrm{~s}$. In MH, $T_{1}$ is set to $1000 \mathrm{~s}$ and $T_{i}$ is set to $60 \mathrm{~s}$. Note that if $\mathrm{MH}$ finds a solution in less than $1000 \mathrm{~s}$ then it is the solution returned by ILP, and, hence, guaranteed to be optimal.

Tables 2, 3 and 4 present the detailed computational results of our four approaches, viz. ABC, IWO, ILP and MH, and, compare these results with those of five heuristic approaches, viz. H1, H2, H3, H4 and H5 (Nakkala and Singh, 2020). These five heuristic approaches are the only approaches available in the literature for MWDDS. These tables report the average solution quality on each group of 10 instances obtained by various approaches under the columns named after respective approaches. These tables also report the average execution times in seconds on each group of 10 instances for ABC, IWO, ILP and MH under the columns named after respective approaches with subscript $E T$, i.e., columns entitled $\mathrm{ABC}_{E T}$, $\mathrm{IWO}_{E T}$, ILP $E T, \mathrm{MH}_{E T}$. Execution times of $\mathrm{H} 1, \mathrm{H} 2, \mathrm{H} 3, \mathrm{H} 4$ and $\mathrm{H} 5$ being negligible will not be reported. Table 2 present the detailed results on UDG instances, Table 3 on Type-I instances and Table 4 on Type-II instances. Table 5 present the summary of comparisons among our 4 proposed approaches in terms of number of groups of instances in each dataset and overall on which an approach mentioned in the left performs better than (B) or same as (E) or worse than the approach mentioned at the top. From these tables, following observations can be made:
- As expected, all the four proposed approaches performed better than the five heuristics available in the literature on all the groups of instances in all the three datasets.

- IWO performed better than ABC in general, and the performance gap between them widens as the instance size increases.

- Barring 10 groups of instances in Type I dataset, where ILP could not finish in allotted time of $3600 \mathrm{~s}$ on some instances, it performed as good as or better than IWO and $\mathrm{ABC}$ both.

- $\mathrm{MH}$ performed as good as or better than IWO and ABC both except for 2 groups of instances in Type I dataset where $A B C$ performed better. It is to be noted that $\mathrm{MH}$ will always perform as good as IWO due to its very design where IWO is one component.

- All UDG and Type II instances were solved to optimality by ILP and $\mathrm{MH}$ both with maximum execution time of around $100 \mathrm{~s}$. Moreover, ILP and MH are several times faster than IWO and ABC on these two datasets.

- MH performed as good as or better than ILP on all groups of instances except for one group of instances in Type I dataset. $\mathrm{MH}$ can perform worse than ILP only on those instances where ILP can find a better solution after $T_{1}$ amount of time in comparison to the solution that it got within $T_{1}$ amount time.

- $\mathrm{MH}$ never required the maximum allotted time of $3600 \mathrm{~s}$. It has terminated before that time in all the cases.

- Among the 4 methods that we have proposed, MH has performed the best. This is not surprising as for different dominating set variants, matheuristic methods are currently the best performing methods, e.g. Albuquerque and Vidal (2018), Pinacho-Davidson et al. (2019), and Nakkala et al. (2021). These matheuristic approaches not only provide solutions of better quality, but are also faster than several metaheuristic approaches for the same problem in most cases.

To determine the impact of parameter settings on the quality of solutions returned by ABC and IWO, for each of their parameters, we have evaluated the performance of these two approaches on three parameter values, viz. the original value, one lower value and one higher value. In all these experiments, all parameters other than the one whose performance is evaluated are set to their original values as reported previously. This has been done one-by-one for all parameters. For this performance evaluation, we have chosen three instance groups, viz. a Type-I instance group with 800 vertices and 5000 arcs, a Type-II instance group with 800 vertices and 10000 arcs, and a Type II instance group with 1000 vertices and 10000 arcs. Performance is reported as sum of average solution quality on each of these three groups. Table 6 reports the results of these experiments. From this table, it can be clearly seen that each of our chosen parameter values provide better results for both $A B C$ and IWO in comparison to tested values on the lower and the higher sides. 
Table 3

Comparison of H1, H2, H3, H4, H5, ABC, IWO, ILP, and MH on Type-I dataset.

\begin{tabular}{|c|c|c|c|c|c|c|c|c|c|c|c|c|c|c|}
\hline$|V|$ & $|A|$ & $\mathrm{H} 1$ & $\mathrm{H} 2$ & H3 & $\mathrm{H} 4$ & H5 & $\mathrm{ABC}$ & $\mathrm{ABC}_{E T}$ & IWO & $\mathrm{IWO}_{E T}$ & ILP & $\mathrm{ILP}_{E T}$ & MH & $\mathrm{MH}_{E T}$ \\
\hline 50 & 50 & 750.3 & 747.5 & 747.5 & 744.7 & 744.7 & 737.6 & 1.3 & 737.6 & 1.2 & 737.6 & 0.0 & 737.6 & 0.0 \\
\hline 50 & 100 & 590.1 & 577.9 & 577.9 & 574.6 & 574.6 & 564.8 & 2.1 & 564.8 & 2.6 & 564.8 & 0.0 & 564.8 & 0.0 \\
\hline 50 & 250 & 322.1 & 322.1 & 323.2 & 318.8 & 318.8 & 300.2 & 3.4 & 300.2 & 3.7 & 300.2 & 0.0 & 300.2 & 0.0 \\
\hline 50 & 500 & 191.6 & 191.6 & 195.7 & 191.6 & 195.7 & 176.0 & 3.5 & 176.0 & 4.1 & 176.0 & 0.0 & 176.0 & 0.0 \\
\hline 50 & 750 & 139.5 & 139.5 & 139.5 & 139.5 & 139.5 & 124.1 & 4.0 & 124.1 & 4.3 & 124.1 & 0.0 & 124.1 & 0.0 \\
\hline 50 & 1000 & 99.8 & 99.8 & 99.8 & 99.8 & 99.8 & 93.2 & 3.7 & 93.2 & 4.4 & 93.2 & 0.0 & 93.2 & 0.0 \\
\hline 100 & 100 & 1492.7 & 1481.1 & 1478.5 & 1485.8 & 1485.8 & 1461.6 & 3.0 & 1461.6 & 3.6 & 1461.6 & 0.0 & 1461.6 & 0.0 \\
\hline 100 & 250 & 1027.2 & 1025.5 & 1025.5 & 1041.4 & 1041.4 & 978.3 & 5.9 & 978.3 & 6.7 & 978.3 & 0.0 & 978.3 & 0.0 \\
\hline 100 & 500 & 674.8 & 668.4 & 668.4 & 667.0 & 667.0 & 618.3 & 8.5 & 617.6 & 9.2 & 617.6 & 0.1 & 617.6 & 0.1 \\
\hline 100 & 750 & 483.8 & 486.4 & 486.4 & 488.0 & 488.0 & 448.2 & 10.1 & 448.2 & 10.9 & 448.2 & 0.1 & 448.2 & 0.1 \\
\hline 100 & 1000 & 398.0 & 398.0 & 398.0 & 398.0 & 398.0 & 360.7 & 11.5 & 360.7 & 12.3 & 360.7 & 0.2 & 360.7 & 0.2 \\
\hline 100 & 2000 & 217.1 & 217.1 & 217.1 & 217.1 & 217.1 & 199.4 & 12.8 & 199.4 & 12.5 & 199.4 & 0.3 & 199.4 & 0.3 \\
\hline 150 & 150 & 2275.8 & 2260.5 & 2260.7 & 2262.7 & 2262.7 & 2237.4 & 5.2 & 2237.4 & 6.0 & 2237.4 & 0.0 & 2237.4 & 0.0 \\
\hline 150 & 250 & 1909.8 & 1880.4 & 1876.5 & 1871.8 & 1874.3 & 1821.6 & 8.2 & 1821.6 & 9.0 & 1821.6 & 0.0 & 1821.6 & 0.0 \\
\hline 150 & 500 & 1287.8 & 1278.9 & 1278.9 & 1273.6 & 1273.6 & 1222.4 & 12.2 & 1222.2 & 12.6 & 1222.1 & 0.1 & 1222.1 & 0.1 \\
\hline 150 & 750 & 1023.7 & 1015.7 & 1015.7 & 998.8 & 998.8 & 927.5 & 14.3 & 927.5 & 15.1 & 927.5 & 0.2 & 927.5 & 0.2 \\
\hline 150 & 1000 & 829.3 & 829.3 & 838.3 & 826.2 & 835.2 & 751.5 & 17.2 & 751.5 & 17.8 & 751.5 & 1.2 & 751.5 & 1.2 \\
\hline 150 & 2000 & 476.1 & 476.1 & 476.1 & 476.1 & 476.1 & 425.5 & 24.0 & 425.4 & 23.9 & 425.4 & 4.7 & 425.4 & 4.6 \\
\hline 150 & 3000 & 345.3 & 345.3 & 345.3 & 345.3 & 345.3 & 306.0 & 27.5 & 306.0 & 26.8 & 306.0 & 10.7 & 306.0 & 10.6 \\
\hline 200 & 250 & 2833.8 & 2808.9 & 2811.8 & 2802.3 & 2805.2 & 2748.6 & 11.2 & 2748.0 & 10.8 & 2748.0 & 0.0 & 2748.0 & 0.0 \\
\hline 200 & 500 & 2065.7 & 2062.8 & 2062.8 & 2049.4 & 2049.3 & 1958.8 & 16.1 & 1958.3 & 16.6 & 1957.8 & 0.0 & 1957.8 & 0.0 \\
\hline 200 & 750 & 1646.9 & 1629.0 & 1625.3 & 1624.4 & 1624.8 & 1530.2 & 20.6 & 1528.0 & 21.0 & 1527.8 & 0.2 & 1527.8 & 0.2 \\
\hline 200 & 1000 & 1336.9 & 1340.5 & 1342.7 & 1341.1 & 1341.1 & 1226.1 & 20.8 & 1225.9 & 21.7 & 1225.9 & 0.5 & 1225.9 & 0.5 \\
\hline 200 & 2000 & 808.0 & 808.0 & 803.1 & 808.0 & 803.1 & 723.8 & 32.9 & 724.6 & 31.3 & 723.8 & 29.2 & 723.8 & 28.9 \\
\hline 200 & 3000 & 587.4 & 587.4 & 587.4 & 587.4 & 587.4 & 518.6 & 42.4 & 518.6 & 39.7 & 518.6 & 72.0 & 518.6 & 71.7 \\
\hline 250 & 250 & 3709.2 & 3678.5 & 3679.6 & 3664.7 & 3663.3 & 3641.7 & 11.4 & 3641.9 & 10.7 & 3641.5 & 0.0 & 3641.5 & 0.0 \\
\hline 250 & 500 & 2943.3 & 2910.5 & 2909.1 & 2906.4 & 2906.0 & 2797.1 & 20.7 & 2797.0 & 20.3 & 2796.9 & 0.0 & 2796.9 & 0.0 \\
\hline 250 & 750 & 2369.7 & 2365.2 & 2365.2 & 2382.1 & 2382.1 & 2234.4 & 25.7 & 2233.6 & 25.3 & 2231.4 & 0.1 & 2231.4 & 0.1 \\
\hline 250 & 1000 & 1956.7 & 1965.5 & 1967.5 & 1942.4 & 1939.6 & 1816.2 & 28.2 & 1815.8 & 31.3 & 1815.1 & 0.4 & 1815.1 & 0.4 \\
\hline 250 & 2000 & 1197.4 & 1197.4 & 1197.1 & 1197.4 & 1197.1 & 1073.9 & 41.7 & 1074.5 & 37.1 & 1073.9 & 85.9 & 1073.9 & 85.1 \\
\hline 250 & 3000 & 884.1 & 884.1 & 884.1 & 884.1 & 884.1 & 784.7 & 56.3 & 785.8 & 47.5 & 784.7 & 773.2 & 784.7 & 516.8 \\
\hline 250 & 5000 & 577.4 & 577.4 & 578.1 & 577.4 & 578.1 & 514.6 & 66.0 & 514.5 & 60.7 & 514.3 & 1670.5 & 514.5 & 795.1 \\
\hline 300 & 300 & 4572.9 & 4530.6 & 4530.6 & 4534.1 & 4534.1 & 4487.6 & 17.7 & 4487.0 & 14.3 & 4486.5 & 0.0 & 4486.5 & 0.0 \\
\hline 300 & 500 & 3850.4 & 3811.0 & 3807.7 & 3810.4 & 3813.1 & 3700.5 & 21.4 & 3698.7 & 23.3 & 3696.9 & 0.0 & 3696.9 & 0.0 \\
\hline 300 & 750 & 3205.9 & 3161.3 & 3168.7 & 3152.0 & 3154.0 & 3010.6 & 34.2 & 3010.4 & 29.2 & 3007.8 & 0.0 & 3007.8 & 0.0 \\
\hline 300 & 1000 & 2667.9 & 2639.7 & 2636.1 & 2635.4 & 2635.4 & 2491.0 & 31.0 & 2490.7 & 34.8 & 2488.1 & 0.4 & 2488.1 & 0.4 \\
\hline 300 & 2000 & 1658.5 & 1658.7 & 1651.5 & 1652.8 & 1648.8 & 1500.4 & 47.6 & 1499.9 & 45.0 & 1499.3 & 118.6 & 1499.3 & 116.3 \\
\hline 300 & 3000 & 1199.4 & 1199.4 & 1195.5 & 1199.4 & 1195.5 & 1095.9 & 67.1 & 1097.6 & 56.8 & 1097.6 & 2332.1 & 1096.5 & 1018.9 \\
\hline 300 & 5000 & 799.0 & 799.0 & 799.0 & 799.0 & 799.0 & 719.6 & 83.5 & 719.6 & 79.1 & 722.4 & 3393.9 & 719.6 & 1079.9 \\
\hline 500 & 500 & 7546.1 & 7485.4 & 7480.4 & 7482.4 & 7482.4 & 7406.3 & 37.8 & 7406.0 & 29.0 & 7399.2 & 0.0 & 7399.2 & 0.0 \\
\hline 500 & 1000 & 5833.9 & 5789.2 & 5786.7 & 5796.7 & 5796.7 & 5597.3 & 55.8 & 5594.8 & 56.7 & 5590.0 & 0.0 & 5590.0 & 0.0 \\
\hline 500 & 2000 & 4001.9 & 3985.7 & 3986.3 & 3973.6 & 3973.6 & 3691.8 & 89.9 & 3689.8 & 90.6 & 3686.0 & 20.6 & 3686.0 & 20.0 \\
\hline 500 & 5000 & 2012.6 & 2012.6 & 2012.6 & 2012.6 & 2012.6 & 1811.7 & 119.9 & 1811.0 & 104.7 & 1825.4 & 3600.3 & 1811.0 & 1146.1 \\
\hline 500 & 10000 & 1200.2 & 1200.2 & 1200.2 & 1200.2 & 1200.2 & 1044.8 & 252.6 & 1048.7 & 183.3 & 1075.4 & 3600.3 & 1048.1 & 1206.5 \\
\hline 800 & 1000 & 11238.7 & 11088.2 & 11087.1 & 11085.4 & 11087.3 & 10947.5 & 75.4 & 10946.0 & 77.2 & 10933.5 & 0.1 & 10933.5 & 0.1 \\
\hline 800 & 2000 & 8442.3 & 8382.7 & 8378.6 & 8350.2 & 8350.2 & 7974.2 & 170.0 & 7968.1 & 161.5 & 7956.8 & 0.7 & 7956.8 & 0.7 \\
\hline 800 & 5000 & 4664.6 & 4639.0 & 4634.8 & 4639.1 & 4636.8 & 4204.1 & 236.4 & 4193.6 & 222.9 & 4210.7 & 3600.2 & 4189.1 & 1574.5 \\
\hline 800 & 10000 & 2697.9 & 2697.9 & 2691.5 & 2697.9 & 2691.5 & 2434.2 & 487.8 & 2432.0 & 240.9 & 2483.7 & 3600.1 & 2431.7 & 1531.1 \\
\hline 1000 & 1000 & 14956.5 & 14847.4 & 14839.8 & 14863.6 & 14863.6 & 14725.8 & 84.0 & 14722.3 & 87.3 & 14708.9 & 0.1 & 14708.9 & 0.1 \\
\hline 1000 & 5000 & 6768.5 & 6814.3 & 6816.6 & 6790.3 & 6792.0 & 6238.5 & 437.0 & 6229.9 & 344.0 & 6228.1 & 3600.2 & 6213.4 & 1801.5 \\
\hline 1000 & 10000 & 4050.6 & 4050.6 & 4057.7 & 4047.0 & 4054.1 & 3647.7 & 462.4 & 3648.3 & 322.0 & 3728.3 & 3600.1 & 3647.3 & 1719.0 \\
\hline 1000 & 15000 & 2945.7 & 2945.7 & 2943.7 & 2945.7 & 2943.7 & 2651.1 & 624.8 & 2650.3 & 456.1 & 2734.9 & 3600.1 & 2650.3 & 1849.6 \\
\hline 1000 & 20000 & 2331.0 & 2331.0 & 2331.0 & 2331.0 & 2331.0 & 2102.5 & 869.6 & 2093.7 & 608.4 & 2215.9 & 3600.0 & 2093.7 & 1973.2 \\
\hline Avg: & & 2530.2 & 2515.6 & 2515.1 & 2513.0 & 2513.1 & 2392.6 & 92.1 & 2391.7 & 72.3 & 2397.9 & 704.2 & 2390.0 & 312.4 \\
\hline
\end{tabular}

Table 4

Comparison of H1, H2, H3, H4, H5, ABC, IWO, ILP, and MH on Type-II dataset.

\begin{tabular}{|c|c|c|c|c|c|c|c|c|c|c|c|c|c|c|}
\hline$|V|$ & $|A|$ & H1 & $\mathrm{H} 2$ & H3 & $\mathrm{H} 4$ & H5 & $\mathrm{ABC}$ & $\mathrm{ABC}_{E T}$ & IWO & $\mathrm{IWO}_{E T}$ & ILP & $\mathrm{ILP}_{E T}$ & $\mathrm{MH}$ & $\mathrm{MH}_{E T}$ \\
\hline 50 & 50 & 100.6 & 101.0 & 100.8 & 103.4 & 103.5 & 100.0 & 1.5 & 100.0 & 1.3 & 100.0 & 0.0 & 100.0 & 0.0 \\
\hline 50 & 100 & 165.9 & 166.7 & 165.8 & 185.6 & 182.2 & 163.4 & 2.3 & 163.4 & 2.8 & 163.4 & 0.0 & 163.4 & 0.0 \\
\hline 50 & 250 & 379.5 & 379.5 & 379.5 & 382.6 & 382.6 & 370.0 & 4.0 & 370.0 & 4.4 & 370.0 & 0.0 & 370.0 & 0.0 \\
\hline 50 & 500 & 521.4 & 521.4 & 521.4 & 521.4 & 521.4 & 495.9 & 4.5 & 495.9 & 4.7 & 495.9 & 0.0 & 495.9 & 0.0 \\
\hline 50 & 750 & 674.3 & 674.3 & 674.3 & 674.3 & 674.3 & 633.0 & 4.5 & 634.8 & 4.5 & 633.0 & 0.0 & 633.0 & 0.0 \\
\hline 50 & 1000 & 618.6 & 618.6 & 618.6 & 618.6 & 618.6 & 598.3 & 4.7 & 598.3 & 3.2 & 598.3 & 0.0 & 598.3 & 0.0 \\
\hline 100 & 100 & 198.9 & 198.6 & 198.4 & 202.3 & 202.3 & 196.6 & 3.2 & 196.6 & 3.8 & 196.6 & 0.0 & 196.6 & 0.0 \\
\hline 100 & 250 & 390.7 & 386.9 & 386.9 & 414.5 & 414.5 & 376.4 & 7.0 & 376.3 & 7.6 & 376.3 & 0.0 & 376.3 & 0.0 \\
\hline 100 & 500 & 712.3 & 710.8 & 711.3 & 723.5 & 724.0 & 686.1 & 10.8 & 686.2 & 10.6 & 686.0 & 0.0 & 686.0 & 0.0 \\
\hline 100 & 750 & 932.9 & 932.9 & 932.9 & 930.7 & 930.7 & 889.3 & 11.8 & 888.6 & 13.1 & 888.6 & 0.0 & 888.6 & 0.0 \\
\hline 100 & 1000 & 1233.9 & 1233.9 & 1233.9 & 1233.9 & 1233.9 & 1148.5 & 15.9 & 1148.5 & 15.1 & 1148.5 & 0.0 & 1148.5 & 0.0 \\
\hline 100 & 2000 & 1743.8 & 1743.8 & 1743.8 & 1743.8 & 1743.8 & 1583.0 & 17.0 & 1583.0 & 16.4 & 1583.0 & 0.0 & 1583.0 & 0.0 \\
\hline 150 & 150 & 307.9 & 304.9 & 305.2 & 308.2 & 308.2 & 302.8 & 5.4 & 302.8 & 6.1 & 302.8 & 0.0 & 302.8 & 0.0 \\
\hline
\end{tabular}


Table 4 (continued).

\begin{tabular}{|c|c|c|c|c|c|c|c|c|c|c|c|c|c|c|}
\hline$|V|$ & $|A|$ & $\mathrm{H} 1$ & $\mathrm{H} 2$ & $\mathrm{H} 3$ & $\mathrm{H} 4$ & H5 & $\mathrm{ABC}$ & $\mathrm{ABC}_{E T}$ & IWO & $\mathrm{IWO}_{E T}$ & ILP & $\mathrm{ILP}_{E T}$ & MH & $\mathrm{MH}_{E T}$ \\
\hline 150 & 250 & 419.9 & 417.7 & 417.4 & 439.8 & 439.4 & 411.4 & 9.3 & 411.4 & 9.9 & 411.4 & 0.0 & 411.4 & 0.0 \\
\hline 150 & 500 & 743.4 & 744.0 & 744.0 & 766.0 & 765.2 & 724.1 & 13.2 & 723.8 & 14.5 & 723.8 & 0.0 & 723.8 & 0.0 \\
\hline 150 & 750 & 1064.2 & 1062.3 & 1060.8 & 1064.9 & 1063.4 & 1018.5 & 18.2 & 1017.4 & 18.1 & 1016.1 & 0.0 & 1016.1 & 0.0 \\
\hline 150 & 1000 & 1358.0 & 1358.0 & 1358.0 & 1358.0 & 1358.0 & 1298.2 & 23.8 & 1297.5 & 21.3 & 1297.5 & 0.0 & 1297.5 & 0.0 \\
\hline 150 & 2000 & 2066.4 & 2066.4 & 2066.4 & 2066.4 & 2066.4 & 1989.5 & 31.5 & 1986.7 & 31.4 & 1986.1 & 0.1 & 1986.1 & 0.1 \\
\hline 150 & 3000 & 2550.3 & 2550.3 & 2550.3 & 2550.3 & 2550.3 & 2432.8 & 38.3 & 2432.8 & 34.7 & 2428.8 & 0.1 & 2428.8 & 0.1 \\
\hline 200 & 250 & 472.7 & 470.3 & 470.9 & 486.8 & 486.8 & 466.6 & 11.3 & 466.6 & 11.5 & 466.6 & 0.0 & 466.6 & 0.0 \\
\hline 200 & 500 & 795.2 & 792.5 & 792.5 & 846.3 & 847.4 & 772.6 & 20.4 & 771.8 & 19.0 & 771.1 & 0.0 & 771.1 & 0.0 \\
\hline 200 & 750 & 1128.4 & 1127.5 & 1127.5 & 1156.3 & 1156.3 & 1076.4 & 22.1 & 1076.0 & 23.2 & 1075.1 & 0.0 & 1075.1 & 0.0 \\
\hline 200 & 1000 & 1404.6 & 1407.3 & 1407.3 & 1402.6 & 1402.6 & 1350.5 & 27.3 & 1349.4 & 25.7 & 1349.0 & 0.0 & 1349.0 & 0.0 \\
\hline 200 & 2000 & 2336.1 & 2336.1 & 2336.1 & 2336.1 & 2336.1 & 2215.1 & 45.0 & 2212.7 & 39.1 & 2211.6 & 0.1 & 2211.6 & 0.1 \\
\hline 200 & 3000 & 2999.0 & 2999.0 & 2999.0 & 2999.0 & 2999.0 & 2851.9 & 53.3 & 2851.9 & 50.7 & 2851.4 & 0.1 & 2851.4 & 0.1 \\
\hline 250 & 250 & 498.7 & 496.0 & 497.4 & 502.5 & 502.4 & 492.7 & 11.2 & 492.7 & 10.9 & 492.7 & 0.0 & 492.7 & 0.0 \\
\hline 250 & 500 & 850.7 & 849.6 & 849.2 & 895.3 & 893.9 & 832.3 & 24.9 & 832.1 & 22.1 & 832.1 & 0.0 & 832.1 & 0.0 \\
\hline 250 & 750 & 1138.5 & 1135.7 & 1135.3 & 1181.7 & 1182.6 & 1107.7 & 28.2 & 1107.3 & 27.2 & 1106.6 & 0.0 & 1106.6 & 0.0 \\
\hline 250 & 1000 & 1511.1 & 1510.1 & 1510.1 & 1551.8 & 1551.3 & 1445.5 & 34.5 & 1443.0 & 32.6 & 1442.5 & 0.0 & 1442.5 & 0.0 \\
\hline 250 & 2000 & 2505.3 & 2505.3 & 2505.3 & 2505.3 & 2505.3 & 2397.7 & 57.0 & 2395.4 & 45.1 & 2389.4 & 0.1 & 2389.4 & 0.1 \\
\hline 250 & 3000 & 3568.3 & 3568.3 & 3568.3 & 3568.3 & 3568.3 & 3267.4 & 61.1 & 3264.7 & 59.4 & 3264.5 & 0.1 & 3264.5 & 0.1 \\
\hline 250 & 5000 & 4633.5 & 4633.5 & 4633.5 & 4633.5 & 4633.5 & 4379.3 & 91.8 & 4373.4 & 81.8 & 4373.4 & 0.2 & 4373.4 & 0.2 \\
\hline 300 & 300 & 629.1 & 625.7 & 626.2 & 632.8 & 632.8 & 621.6 & 15.6 & 621.6 & 14.1 & 621.6 & 0.0 & 621.6 & 0.0 \\
\hline 300 & 500 & 891.8 & 888.0 & 887.4 & 941.2 & 941.7 & 871.4 & 25.0 & 871.0 & 25.8 & 870.3 & 0.0 & 870.3 & 0.0 \\
\hline 300 & 750 & 1256.0 & 1250.7 & 1249.8 & 1296.0 & 1296.5 & 1216.0 & 32.3 & 1215.8 & 32.8 & 1215.4 & 0.0 & 1215.4 & 0.0 \\
\hline 300 & 1000 & 1630.4 & 1625.8 & 1627.9 & 1665.4 & 1663.5 & 1556.6 & 36.4 & 1555.5 & 36.8 & 1552.7 & 0.0 & 1552.7 & 0.0 \\
\hline 300 & 2000 & 2657.2 & 2657.3 & 2657.3 & 2672.2 & 2672.2 & 2531.3 & 56.3 & 2527.1 & 51.2 & 2523.2 & 0.1 & 2523.2 & 0.1 \\
\hline 300 & 3000 & 3717.0 & 3717.0 & 3719.1 & 3717.0 & 3719.1 & 3536.5 & 75.1 & 3534.2 & 74.0 & 3520.9 & 0.2 & 3520.9 & 0.1 \\
\hline 300 & 5000 & 5115.8 & 5115.8 & 5115.8 & 5115.8 & 5115.8 & 4797.3 & 104.6 & 4788.1 & 97.3 & 4776.9 & 0.2 & 4776.9 & 0.2 \\
\hline 500 & 500 & 1022.5 & 1018.2 & 1017.0 & 1034.0 & 1033.8 & 1011.2 & 36.1 & 1011.3 & 31.6 & 1011.0 & 0.0 & 1011.0 & 0.0 \\
\hline 500 & 1000 & 1711.4 & 1699.2 & 1699.9 & 1800.7 & 1802.5 & 1675.0 & 62.2 & 1674.5 & 59.8 & 1672.3 & 0.0 & 1672.3 & 0.0 \\
\hline 500 & 2000 & 3038.9 & 3036.9 & 3036.9 & 3111.0 & 3114.9 & 2915.3 & 104.3 & 2915.4 & 94.0 & 2904.0 & 0.0 & 2904.0 & 0.0 \\
\hline 500 & 5000 & 6116.7 & 6116.7 & 6118.6 & 6116.7 & 6118.6 & 5792.5 & 148.4 & 5789.3 & 127.9 & 5769.9 & 0.4 & 5769.9 & 0.4 \\
\hline 500 & 10000 & 9761.6 & 9761.6 & 9761.6 & 9761.6 & 9761.6 & 9084.1 & 251.8 & 9063.2 & 243.7 & 9043.2 & 0.7 & 9043.2 & 0.7 \\
\hline 800 & 1000 & 1891.8 & 1880.4 & 1879.7 & 1945.6 & 1945.2 & 1864.0 & 91.6 & 1863.1 & 86.2 & 1860.5 & 0.1 & 1860.5 & 0.1 \\
\hline 800 & 2000 & 3342.2 & 3339.7 & 3340.4 & 3493.5 & 3494.7 & 3247.1 & 165.1 & 3243.7 & 158.6 & 3233.0 & 0.1 & 3233.0 & 0.1 \\
\hline 800 & 5000 & 6905.2 & 6909.9 & 6909.9 & 6917.3 & 6917.3 & 6605.8 & 273.5 & 6604.9 & 311.9 & 6561.7 & 0.4 & 6561.7 & 0.4 \\
\hline 800 & 10000 & 11541.3 & 11541.3 & 11541.3 & 11541.3 & 11541.3 & 10802.6 & 407.3 & 10778.1 & 376.8 & 10741.5 & 1.5 & 10741.5 & 1.5 \\
\hline 1000 & 1000 & 2023.7 & 2012.2 & 2012.4 & 2041.1 & 2040.4 & 2001.5 & 90.7 & 2001.3 & 91.8 & 2000.6 & 0.1 & 2000.6 & 0.1 \\
\hline 1000 & 5000 & 7365.4 & 7354.1 & 7354.6 & 7449.7 & 7450.3 & 7030.7 & 375.6 & 7027.8 & 408.6 & 6975.0 & 0.5 & 6975.0 & 0.5 \\
\hline 1000 & 10000 & 12489.7 & 12489.7 & 12485.1 & 12475.4 & 12470.8 & 11745.2 & 488.6 & 11702.7 & 602.8 & 11642.5 & 7.1 & 11642.5 & 6.8 \\
\hline 1000 & 15000 & 16447.3 & 16447.3 & 16447.3 & 16447.3 & 16447.3 & 15414.2 & 618.3 & 15374.2 & 492.1 & 15267.1 & 104.6 & 15267.1 & 99.0 \\
\hline 1000 & 20000 & 19694.3 & 19694.3 & 19694.3 & 19694.3 & 19694.3 & 18278.0 & 835.8 & 18240.6 & 693.6 & 18172.5 & 95.5 & 18172.5 & 90.9 \\
\hline Avg: & & 3005.2 & 3003.5 & 3003.5 & 3023.1 & 3023.1 & 2842.9 & 94.6 & 2838.8 & 90.3 & 2829.6 & 4.1 & 2829.6 & 3.9 \\
\hline
\end{tabular}

Table 5

Performance comparison summary of 4 proposed approaches.

\begin{tabular}{|c|c|c|c|c|c|c|c|c|c|c|c|c|c|}
\hline \multirow[t]{2}{*}{ Dataset } & \multirow[t]{2}{*}{ Method } & \multicolumn{3}{|c|}{$\mathrm{ABC}$} & \multicolumn{3}{|c|}{ IWO } & \multicolumn{3}{|l|}{ ILP } & \multicolumn{3}{|c|}{$\mathrm{MH}$} \\
\hline & & B & $\mathrm{E}$ & W & B & $\mathrm{E}$ & W & B & $\mathrm{E}$ & W & B & $\mathrm{E}$ & $\mathrm{W}$ \\
\hline \multirow{4}{*}{ UDG } & $\mathrm{ABC}$ & - & - & - & 2 & 3 & 7 & 0 & 3 & 9 & 0 & 3 & 9 \\
\hline & IWO & 7 & 3 & 2 & - & - & - & 0 & 3 & 9 & 0 & 3 & 9 \\
\hline & ILP & 9 & 3 & 0 & 9 & 3 & 0 & - & - & - & 0 & 12 & 0 \\
\hline & $\mathrm{MH}$ & 9 & 3 & 0 & 9 & 3 & 0 & 0 & 12 & 0 & - & - & - \\
\hline \multirow{4}{*}{ Type-I } & $\mathrm{ABC}$ & - & - & - & 7 & 18 & 28 & 9 & 20 & 24 & 2 & 21 & 30 \\
\hline & IWO & 28 & 18 & 7 & - & - & - & 8 & 22 & 23 & 0 & 26 & 27 \\
\hline & ILP & 24 & 20 & 9 & 23 & 22 & 8 & - & - & - & 1 & 42 & 10 \\
\hline & $\mathrm{MH}$ & 30 & 21 & 2 & 27 & 26 & 0 & 10 & 42 & 1 & - & - & - \\
\hline \multirow{4}{*}{ Type-II } & $\mathrm{ABC}$ & - & - & - & 4 & 15 & 34 & 0 & 14 & 39 & 0 & 14 & 39 \\
\hline & IWO & 34 & 15 & 4 & - & - & - & 0 & 19 & 34 & 0 & 19 & 34 \\
\hline & ILP & 39 & 14 & 0 & 34 & 19 & 0 & - & - & - & 0 & 53 & 0 \\
\hline & MH & 39 & 14 & 0 & 34 & 19 & 0 & 0 & 53 & 0 & - & - & - \\
\hline \multirow{4}{*}{ Overall } & $\mathrm{ABC}$ & - & - & - & 13 & 36 & 69 & 9 & 37 & 72 & 2 & 38 & 78 \\
\hline & IWO & 69 & 36 & 13 & - & - & - & 8 & 44 & 66 & 0 & 48 & 70 \\
\hline & ILP & 72 & 37 & 9 & 66 & 44 & 8 & - & - & - & 1 & 107 & 10 \\
\hline & $\mathrm{MH}$ & 78 & 38 & 2 & 70 & 48 & 0 & 10 & 107 & 1 & - & - & - \\
\hline
\end{tabular}

\section{Conclusions}

Minimum weight dominating set problem (MWDS) and minimum directed dominating set problem (MDDS) are well-known variants of minimum dominating set problem and many approaches are available in the literature to solve them. However, the minimum weight directed dominating set problem (MWDDS) that has several real-world applications and can be considered as a generalization of MWDS and MDDS both has not received much attention. Only five problem specific heuristics were available in the literature (Nakkala and Singh, 2020), and this problem has neither been studied in the literature from metaheuristic perspective nor from integer linear programming perspective nor from matheuristic perspectives. This paper proposed two swarm intelligence based metaheuristics, viz. ABC and IWO, an ILP approach and a matheuristic approach $\mathrm{MH}$ and experimentally evaluated their performance on three datasets derived from respective datasets of MWDS. All the four approaches performed better than five problem specific heuristics. IWO performed better than ABC. MH and 
Table 6

Influence of parameter settings on solution quality returned by $\mathrm{ABC}$ and IWO.

\begin{tabular}{|c|c|c|c|c|c|c|c|}
\hline \multicolumn{4}{|l|}{$\mathrm{ABC}$} & \multicolumn{4}{|l|}{ IWO } \\
\hline Parameter & Tested values & Corresponding results & Best value & Parameter & Tested values & Corresponding results & Best value \\
\hline$E_{n}$ & $\{50,75,100\}$ & $\{26763.2,26751.9,26770.6\}$ & 75 & $n_{\text {init }}$ & $\{250,300,350\}$ & $\{26723.6,26674.4,26746.1\}$ & 300 \\
\hline$O_{n}$ & $\{100,125,150\}$ & $\{26784.7,26751.9,26786.3\}$ & 125 & $x_{\min }$ & $\{0,1,2\}$ & $\{26746.7,26674.4,26711.1\}$ & 1 \\
\hline limit $_{\text {non_imp }}$ & $\{500,600,700\}$ & $\{26776.0,26751.9,26780.3\}$ & 600 & $x_{\max }$ & $\{2,3,4\}$ & $\{26693.6,26674.4,26718.7\}$ & 3 \\
\hline$R$ & $\{0.5,0.6,0.7\}$ & $\{26791.3,26751.9,26787.2\}$ & 0.6 & $R$ & $\{0.5,0.6,0.7\}$ & $\{26754.7,26674.4,26747.4\}$ & 0.6 \\
\hline$I_{\text {update }}$ & $\{7,8,9\}$ & $\{26774.2,26751.9,26769.9\}$ & 8 & $I_{\text {update }}$ & $\{4,5,6\}$ & $\{26708.8,26674.4,26717.5\}$ & 5 \\
\hline$P_{o l}$ & $\{0.7,0.8,0.9\}$ & $\{26780.7,26751.9,26772.3\}$ & 0.8 & $n_{\max }$ & $\{300,350,400\}$ & $\{26757.0,26674.4,26716.3\}$ & 350 \\
\hline$P_{r}$ & $\{0.2,0.3,0.4\}$ & $\{26775.2,26751.9,26769.6\}$ & 0.3 & $P_{r}$ & $\{0.2,0.3,0.4\}$ & $\{26745.3,26674.4,26736.1\}$ & 0.3 \\
\hline
\end{tabular}

ILP performed better than both $\mathrm{ABC}$ and IWO. MH performed better than ILP, and, hence, it is the overall best approach to solve MWDDS.

As a future work, we intend to work on few more metaheuristic and matheuristic approaches for MWDDS. The adaptive mechanism used here for selecting a heuristic can be used for other problems also where more than one problem-specific heuristics are available. Success of $\mathrm{MH}$ again stresses the effectiveness of matheuristics in solving the dominating set variants (Albuquerque and Vidal, 2018; Pinacho-Davidson et al., 2019; Nakkala et al., 2021). Hence, new matheuristic approaches need to be explored for solving different dominating set variants and other related problems like vertex cover and independent set variants.

\section{CRediT authorship contribution statement}

Mallikarjun Rao Nakkala: Conceptualization, Methodology, Software, Formal analysis, Investigation, Writing - original draft, Visualization. Alok Singh: Supervision, Conceptualization, Methodology, Formal analysis, Investigation, Validation, Writing - review \& editing, Resources. André Rossi: Conceptualization, Methodology, Software, Validation, Formal analysis, Writing - original draft, Writing - review \& editing.

\section{Declaration of competing interest}

The authors declare that they have no known competing financial interests or personal relationships that could have appeared to influence the work reported in this paper.

\section{Acknowledgments}

Authors are grateful to the associate editor and the three anonymous reviewers for their valuable comments and suggestions which helped in improving the quality of this manuscript. The first author acknowledges the financial support received from the Council of Scientific \& Industrial Research (CSIR), Government of India in the form of a senior research fellowship.

\section{References}

Akay, B., Karaboga, D., Gorkemli, B., Kaya, E., 2021. A survey on the artificial bee colony algorithm variants for binary, integer and mixed integer programming problems. Appl. Soft Comput. 106, 107351.

Albuquerque, M., Vidal, T., 2018. An efficient matheuristic for the minimum-weight dominating set problem. Appl. Soft Comput. 72, 527-538.

Alshamiri, A.K., Singh, A., Surampudi, B.R., 2016. Artificial bee colony algorithm for clustering: an extreme learning approach. Soft Comput. 20 (8), 3163-3176.

Alshamiri, A.K., Singh, A., Surampudi, B.R., 2018. Two swarm intelligence approaches for tuning extreme learning machine. Int. J. Mach. Learn. Cybern. 9 (8), 1271-1283.

Chaurasia, S.N., Singh, A., 2015a. A hybrid evolutionary algorithm with guided mutation for minimum weight dominating set. Appl. Intell. 43 (3), 512-529.

Chaurasia, S.N., Singh, A., 2015b. A hybrid swarm intelligence approach to the registration area planning problem. Inform. Sci. 302, 50-69.

Garey, M., Johnson, D.S., 1979. Computers and Intractability: A Guide to the Theory of NP-Completeness. W. H. Freeman.

Ghoshal, J., Laskar, R., Pillone, D., 1998. Topics on domination in directed graphs. In: Haynes, T., Hedetniemi, S., Slater, P. (Eds.), Domination in Graphs: Advanced Topics. Marcel Dekker, New York, pp. 401-438.
Habibulla, Y., Zhao, J.-H., Zhou, H.-J., 2015. The directed dominating set problem: Generalized leaf removal and belief propagation. In: International Workshop on Frontiers in Algorithmics. Springer, pp. 78-88.

Jayalakshmi, B., Singh, A., 2017. A hybrid artificial bee colony algorithm for the p-median problem with positive/negative weights. Opsearch 54 (1), 67-93.

Jovanovic, R., Tuba, M., Simian, D., 2010. Ant colony optimization applied to minimum weight dominating set problem. In: Proceedings of the 12th WSEAS International Conference on Automatic Control, Modelling \& Simulation. In: ACMOS'10, World Scientific and Engineering Academy and Society (WSEAS), Stevens Point, Wisconsin, USA, pp. 322-326.

Karaboga, D., 2005. An idea based on honey bee swarm for numerical optimization. Tech. rep, Technical Report TR06, Computer Engineering Department, Erciyes University, Turkey.

Koltun, V., Papadimitriou, C.H., 2005. Approximately dominating representatives. In: Eiter, T., Libkin, L. (Eds.), Proceedings of the International Conference on Database Theory. ICDT 2005, In: Lecture Notes in Computer Science, vol. 3363, Springer, Berlin, Heidelberg, pp. 204-214.

Lin, G., Guan, J., 2018. A binary particle swarm optimization for the minimum weight dominating set problem. J. Comput. Sci. Tech. 33 (2), 305-322.

Lin, G., Zhu, W., Ali, M., 2016. An effective hybrid memetic algorithm for the minimum weight dominating set problem. IEEE Trans. Evol. Comput. 20 (6), 892-907.

Liu, B., Nie, L., 2021. Gradient based invasive weed optimization algorithm for the training of deep neural network. Multimedia Tools Appl. https://doi.org/10.1007/s11042-020-10495-3.

Liu, Y., Slotine, J., Barabási, A., 2013. Observability of complex systems. In: Proceedings of the National Academy of Sciences, Vol. 110. National Academy of Sciences, USA, USA, pp. 2460-2465.

Mehrabian, A.R., Lucas, C., 2006. A novel numerical optimization algorithm inspired from weed colonization. Ecol. Inform. 1 (4), 355-366.

Molnár Jr., F., Sreenivasan, S., Szymanski, B., Korniss, K., 2013. Minimum dominating sets in scale-free network ensembles. Sci. Rep. 3, 1736.

Nakkala, M.R., Singh, A., 2020. Heuristics for minimum weight directed dominating set problem. In: Futuristic Trends in Networks and Computing Technologies, Communications in Computer and Information Science. 1206, Springer, pp. 494-507.

Nakkala, M.R., Singh, A., Rossi, A., 2021. Multi-start iterated local search, exact and matheuristic approaches for minimum capacitated dominating set problem. Appl. Soft Comput. 108, 107437.

Nitash, C., Singh, A., 2014. An artificial bee colony algorithm for minimum weight dominating set. In: 2014 IEEE Symposium on Swarm Intelligence. IEEE, pp. 1-7.

Pandiri, V., Singh, A., 2018. A hyper-heuristic based artificial bee colony algorithm for k-interconnected multi-depot multi-traveling salesman problem. Inform. Sci. 463-464, 261-281.

Pandiri, V., Singh, A., 2019. An artificial bee colony algorithm with variable degree of perturbation for the generalized covering traveling salesman problem. Appl. Soft Comput. 78, 481-495.

Pandiri, V., Singh, A., Rossi, A., 2020. Two hybrid metaheuristic approaches for the covering salesman problem. Neural Comput. Appl. 32 (19), 15643-15663.

Pang, C., Zhang, R., Zhang, Q., Wang, J., 2010. Dominating sets in directed graphs. Inforation Sci. 180, 3647-3652.

Pinacho-Davidson, P., Bouamama, S., Blum, C., 2019. Application of CMSA to the minimum capacitated dominating set problem. In: Proceedings of the Genetic and Evolutionary Computation Conference 2019. GECCO 2019, ACM, pp. 321-328.

Potluri, A., Singh, A., 2013. Hybrid metaheuristic algorithms for minimum weight dominating set. Appl. Soft Comput. 13 (1), 76-88.

Safari, M.J.S., Mohammadi, B., Kargar, K., 2020. Invasive weed optimization-based adaptive neuro-fuzzy inference system hybrid model for sediment transport with a bed deposit. J. Cleaner Prod. 276, 124267.

Shetgaonkar, S., Singh, A., 2020. Hybridization of artificial bee colony algorithm with estimation of distribution algorithm for minimum weight dominating set problem. In: Proceeding of the Sixth International Conference on ICT for Sustainable Development. ICT4SD 2020, In: Advances in Intelligent Systems and Computing, vol. 1270, Springer, pp. 1-16.

Singh, A., 2009. An artificial bee colony algorithm for the leaf-constrained minimum spanning tree problem. Appl. Soft Comput. 9 (2), 625-631. 
Singh, A., Sundar, S., 2011. An artificial bee colony algorithm for the minimum routing cost spanning tree problem. Soft Comput. 15 (12), 2489-2499.

Takaguchi, T., Hasegawa, T., Yoshida, Y., 2014. Suppressing epidemics on networks by exploiting observer nodes. Phys. Rev. E 90, 012807.

Venkatesh, P., Singh, A., 2015. Two metaheuristic approaches for the multiple traveling salesperson problem. Appl. Soft Comput. 26, 74-89.

Wang, Y., Cai, S., Chen, J., Yin, M., 2018. A fast local search algorithm for minimum weight dominating set problem on massive graphs. In: Proceedings of the TwentySeventh International Joint Conference on Artificial Intelligence. IJCAI 2018, IJCAI, pp. 1514-1522.
Wang, H., Zheng, H., Browne, F., Wang, C., 2014. Minimum dominating sets in cell cycle specific protein interaction networks. In: Proceedings of the Interanational Conference on Bioinformatics and Biomedicine. BIBM 2014, IEEE, pp. 25-30.

Wu, J., 2002. Extended dominating-set-based routing in Ad Hoc wireless networks with unidirectional links. IEEE Trans. Parallel Distrib. Syst. 13, 866-881.

Wuchty, S., 2014. Controllability in protein interaction networks. In: Proceedings of the National Academy of Sciences, Vol. 111. National Academy of Sciences, USA, USA, pp. 7156-7160.

Yang, Y., Wang, J., Motter, A., 2012. Network observability transitions. Phys. Rev. Lett. 90, 258701. 